\title{
Loss of SPARC in bladder cancer enhances carcinogenesis and progression
}

\author{
Neveen Said,, 1,2 Henry F. Frierson, ${ }^{3}$ Marta Sanchez-Carbayo, ${ }^{4}$
} Rolf A. Brekken, ${ }^{5}$ and Dan Theodorescu2,6,7

\begin{abstract}
1Department of Radiation Oncology, 2Department of Urology, and ${ }^{3}$ Department of Pathology, University of Virginia, Charlottesville, Virginia, USA. ${ }^{4}$ Spanish National Cancer Institute (CNIO), Madrid, Spain. ${ }^{5}$ Departments of Surgery and Pharmacology, University of Texas Southwestern Medical Center, Dallas, Texas, USA. ${ }^{6}$ Departments of Surgery and Pharmacology, University of Colorado, Aurora, Colorado, USA.

7University of Colorado Comprehensive Cancer Center, Aurora, Colorado, USA.
\end{abstract}

\begin{abstract}
Secreted protein acidic and rich in cysteine (SPARC) has been implicated in multiple aspects of human cancer. However, its role in bladder carcinogenesis and metastasis are unclear,with some studies suggesting it may be a promoter and others arguing the opposite. Using a chemical carcinogenesis model in Sparc-deficient mice and their wild-type littermates, we found that loss of SPARC accelerated the development of urothelial preneoplasia (atypia and dysplasia), neoplasia, and metastasis and was associated with decreased survival. SPARC reduced carcinogen-induced inflammation and accumulation of reactive oxygen species as well as urothelial cell proliferation. Loss of SPARC was associated with an inflammatory phenotype of tumor-associated macrophages and fibroblasts, with concomitant increased activation of urothelial and stromal NF- $\mathrm{B}$ and AP1 in vivo and in vitro. Syngeneic spontaneous and experimental metastasis models revealed that tumorand stroma-derived SPARC reduced tumor growth and metastasis through inhibition of cancer-associated inflammation and lung colonization. In human bladder tumor tissues, the frequency and intensity of SPARC expression were inversely correlated with disease-specific survival. These results indicate that SPARC is produced by benign and malignant compartments of bladder carcinomas where it functions to suppress bladder carcinogenesis, progression, and metastasis.
\end{abstract}

\section{Introduction}

Bladder cancer is caused primarily by tobacco use and exposure to industrial chemicals (1) and will affect an estimated 73,510 patients and lead to 14,880 deaths in 2012 (2). $N$-nitrosodibutylamine $(\mathrm{BBN})$ was first identified as a bladder carcinogen in rodents (3) and is detected in tobacco smoke and environmental and infectious metabolites (3). Carcinogenesis is a multistep process consisting of initiation, promotion, and progression and is governed by cumulative genetic and epigenetic alterations and microenvironmental cues (4), with inflammation playing an important role as is a tumor promoter via proinflammatory cytokines/chemokines and $\operatorname{ROS}(5,6)$.

Secreted protein acidic and rich in cysteine (SPARC, osteonectin, BM-40) is regulated in tissues undergoing remodeling, during normal development, during tissue repair, and in cancer (reviewed in refs. 7-10). Interest in SPARC has grown in recent years because of its apparent role in regulating tumor growth via its production by both cancer and or stromal cells and based on its interactions with biologically active cytokines/chemokines in the tumor milieu (summarized in refs. 7-10). However, some of the literature appears contradictory regarding how SPARC regulates tumor growth, and many questions remain. For example, increased expression of SPARC is associated with an aggressive tumor phenotype in melanomas and gliomas (reviewed in refs. 7-10), while other studies have reported SPARC as a tumor suppressor whose expression is frequently lost in cancerous compartment due to promoter methylation (8-10). Use of SPARC knockout (Sparc $\left.{ }^{-/-}\right)$ mice revealed that SPARC suppresses syngeneic and oncogene-

Conflict of interest: The authors have declared that no conflict of interest exists. Citation for this article: J Clin Invest. 2013;123(2):751-766. doi:10.1172/JCI64782. driven tumors $(9,11-14)$ through regulation of matrix deposition and through antiinflammatory, antiangiogenic, antiproliferative, and proapoptotic effects, while SPARC has been found to be upregulated in the stroma $(9,15)$. SPARC exerts autocrine and paracrine inhibition of cancer cell proliferation through cell-cycle arrest $(8,9,13,16,17)$ and suppression of survival signaling $(7,11$, $12,18,19)$, cancer cell adhesion, and invasion $(8,11,12,17,18)$.

The role of SPARC in urinary bladder physiology and cancer is also unclear and conflicting. SPARC is expressed in normal murine and human urothelia and suburothelial stroma (20) and in primary cultures of human urothelial cells (21-23). SPARC has been shown to exert an antiadhesive and antiproliferative effect on human and murine urothelial cells (21-23). A few studies have reported expression of SPARC in urothelial tumors, with conflicting results between gene expression profiling and immunolocalization of SPARC protein in tumor tissues. In one study, gene expression profiling of invasive bladder tumors revealed that higher expression of SPARC inversely correlated with prognosis and patient survival (24). Another study (25) showed via immunostaining that SPARC protein was exclusively localized to the desmoplastic stroma within and around the SPARC-negative invasive carcinoma. Additionally, a recent study (26) reported that carcinogenic heavy metals downregulated SPARC expression during malignant transformation of immortalized UROtsa cells. Immunostaining of xenografts of the transformed cells exhibited strong immunoreactivity for the SPARC protein in the host (murine) stromal component surrounding SPARC-negative tumor cells. Furthermore, in bladder cancer cell lines, gene expression profiling of poorly tumorigenic T24 cells and its isogenic metastatic variant T24T revealed an approximately 7.2-fold decrease in SPARC mRNA expression in the invasive T24T (27). 
A

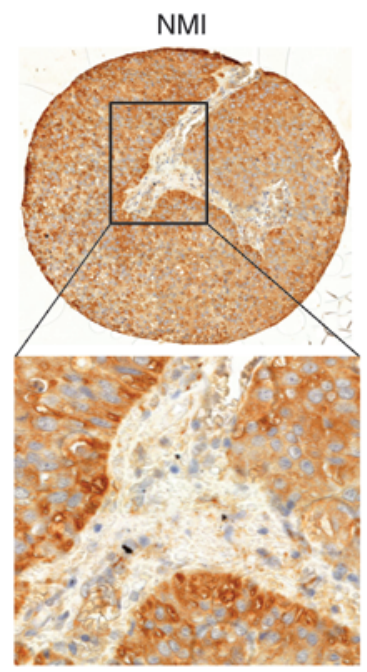

B

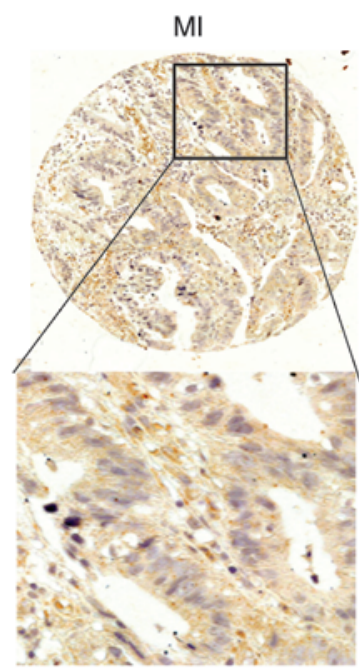

\section{Figure 1}

Expression of SPARC in human bladder cancer tumors. (A and B) IHC staining of SPARC in NMI $(n=92)$ and MI $(n=102)$ tumors showing expression of SPARC in cancerous and stromal cells with an overall decrease in tumor cells in MI disease. Original magnification, $\times 100 ; \times 200$ (inset). (C) Kaplan-Meier curve showing disease-specific survival in high- and low-scoring tumors. Tumors were scored by counting the number of cancer cells expressing the protein or the intensity of SPARC expression (D) as determined by SPARC staining in cancer cells (see Methods) and DSS. Results of log-rank test shown.
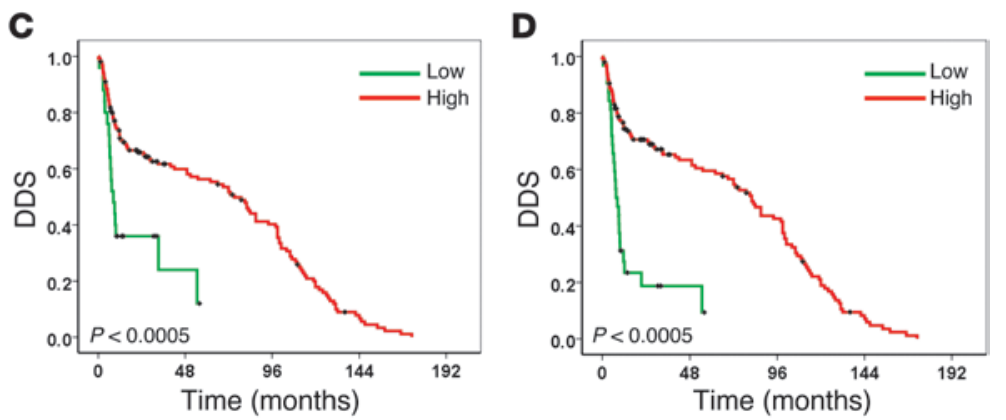

Taken together, the current literature does not provide a unifying picture of the role of SPARC in cancer, nor does it assign the relative contributions of tumor- and stroma-derived SPARC. Herein we address these issues by using urothelial cancer as a model and find that SPARC is produced by benign and malignant compartments of bladder carcinomas, where it functions to suppress bladder carcinogenesis, progression, and metastasis.

\section{Results}

SPARC expression in human bladder cancer is associated with stage and outcome. To determine the relevance of SPARC expression to human disease, we evaluated a human bladder cancer tissue microarray (TMA) $(28,29)$ for SPARC protein expression (Figure 1, A and B) and found that in non-muscle invasive (NMI) disease, SPARC was expressed in the cancerous urothelium and adjacent stroma. In contrast, in muscle invasive (MI) disease, the expression of SPARC in the cancerous tissue was decreased and staining was mainly observed in the tumor-associated stroma. We sought to distinguish the differential compartmentalization of SPARC in human tumors with respect to the frequency of SPARC expression (number of cells expressing the protein) as well as the intensity of expression in cancer cells. SPARC staining was exclusively cytoplasmic in tumor and stromal cells in all the examined cores. The specificity of SPARC immunostaining was confirmed as described in Methods. We used 2 scoring systems, as described in Methods, and determined SPARC tumor cell expression as a function of disease outcome. We found that both the frequency (Figure 1C) and the intensity (Figure 1D) of SPARC staining were positively correlated with disease-specific survival (DSS). However, there was no relationship between the intensity and/or frequency of stromal SPARC expression and DSS.

Loss of SPARC accelerates BBN-induced bladder carcinogenesis and metastasis. To determine the relevance of SPARC in the pathobiology of bladder cancer, we used Sparc ${ }^{-1-}$ mice and their wild-type counterparts $\left(\right.$ Sparc $\left.^{+/+}\right)$in a carcinogen-induced model of bladder cancer, as described in Methods (3), that shares molecular similarities to human disease (30). It is noteworthy to mention that there were no developmental, functional, or histologic differences observed between normal Sparc ${ }^{-/}$and Sparc ${ }^{+/+}$bladders. In both genotypes, the sequence of bladder abnormalities after carcinogen exposure progressed from inflammation with urothelial atypia and dysplasia (IAD) and carcinoma in situ (CIS) to invasive carcinoma (Figure 2, A and B). Sparc ${ }^{-1-}$ mice exhibited accelerated urothelial pathology in all cohorts (Figure 2B). Sparc ${ }^{-1}$ mice exhibited significantly decreased survival compared with their Sparc ${ }^{+/+}$littermates, with a median survival of 42 and 20 weeks for Sparc ${ }^{+/+}$and Sparc ${ }^{/-}$, respectively, and a hazard ratio of 0.0173 and $95 \%$ CI of the ratio 0.004 to 0.07 (Figure 2C). Mortality of both genotypes was primarily due to obstructive uropathy. Loss of SPARC was also associated with pathological changes in the renal urothelium similar to those in the bladder (Figure 2D). Only mice with invasive primary bladder cancers developed metastases mainly to the para-aortic LNs and lungs (Figure 2E), with Sparc ${ }^{-1}$ mice exhibiting a higher incidence of metastases. Macroscopic and microscopic examination of lung lesions with metastases revealed that $\mathrm{Sparc}^{-/}$lungs exhibited greater number and size of metastatic nodules (Figure 2, F and G). In addition, the expression of SPARC protein in bladders exhibited distinctive expression as the 
A

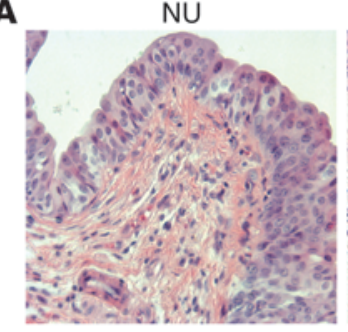

IAD

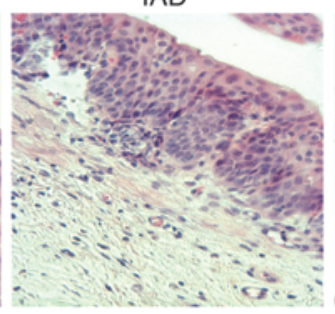

CIS

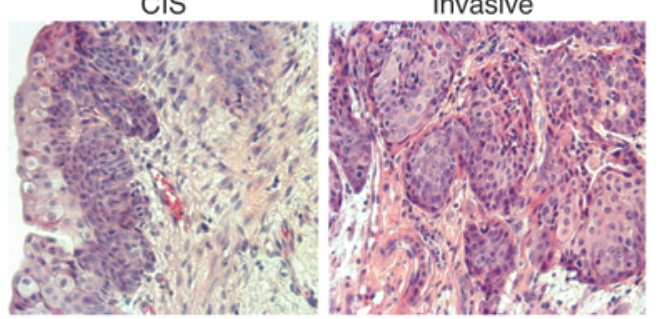

B

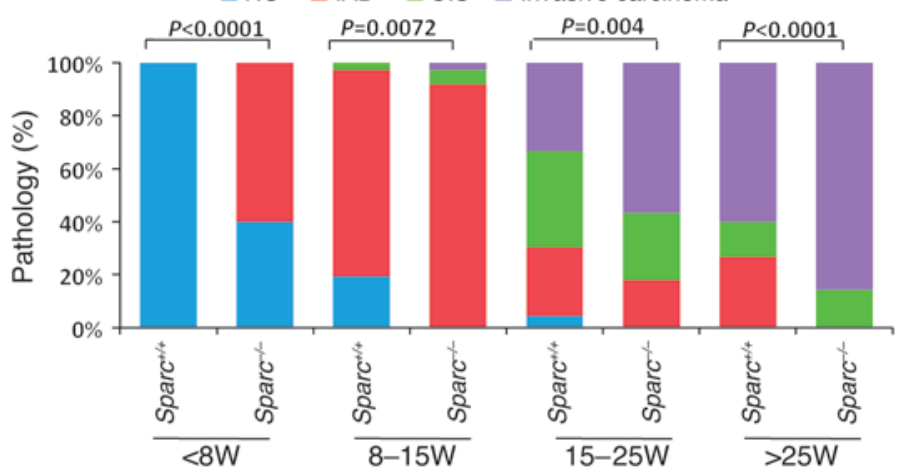

C

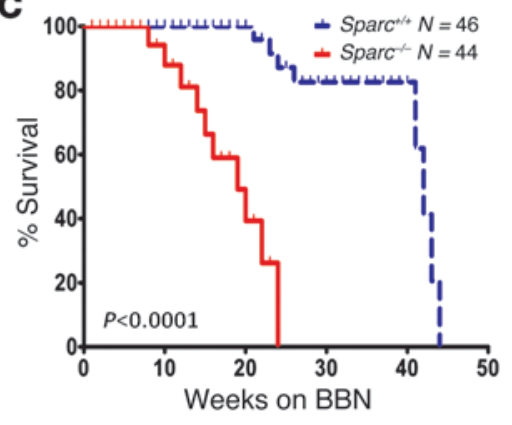

D

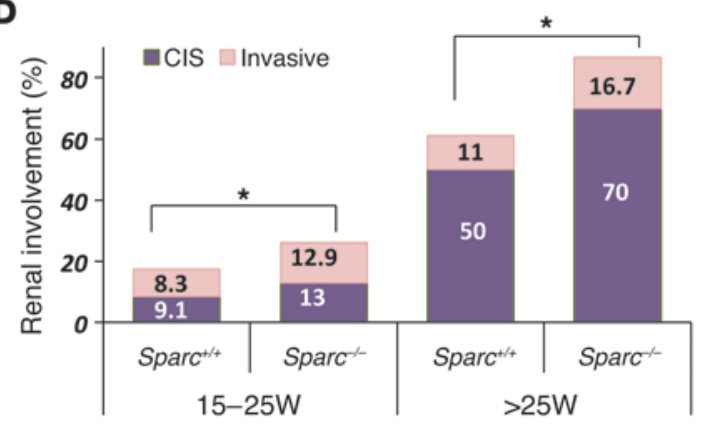

F

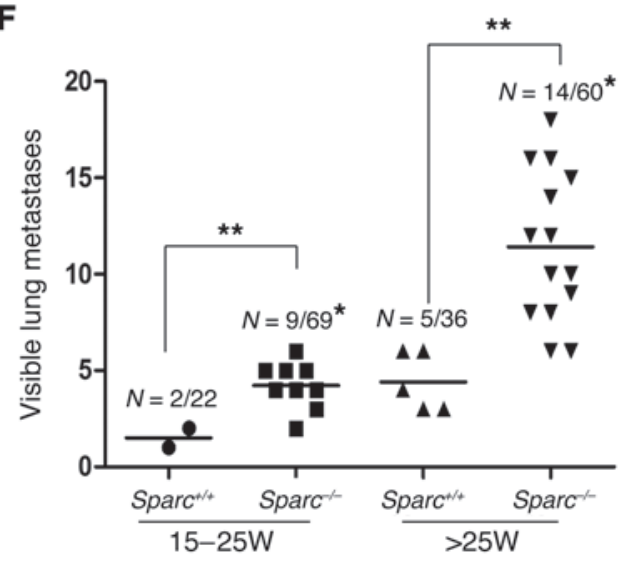

E

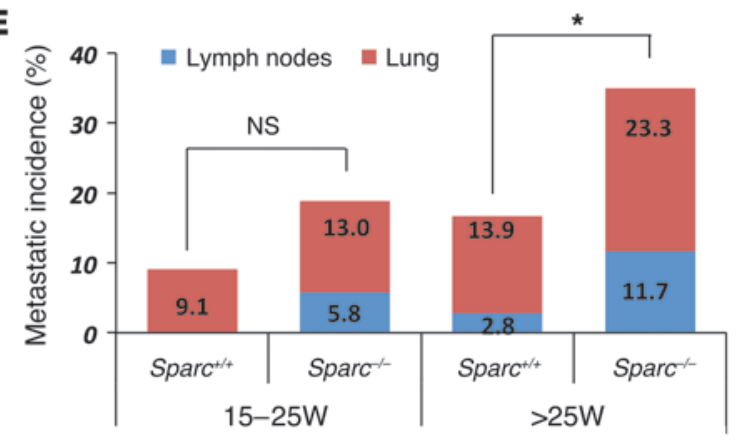

G

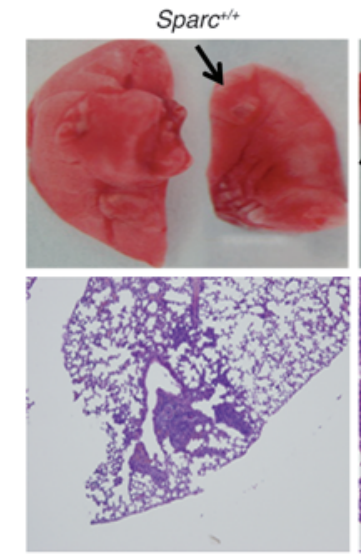

Sparc

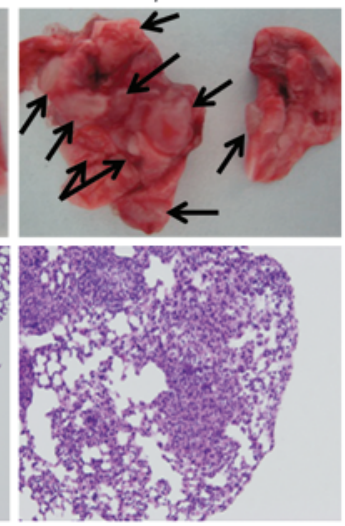

Figure 2

Loss of SPARC accelerates bladder carcinogenesis and progression. (A) Representative H\&E-stained sections of bladder pathology. Original magnification, $\times 200$. (B) Mice were assigned to 4 cohorts as a function of BBN exposure, and the distribution of the ensuing bladder pathology in each cohort is represented in bar graphs showing the percentage of animals $\left(P<0.05, \chi^{2}\right.$ test). (C) Kaplan-Meier curves showing significantly decreased survival in Sparc ${ }^{-/}$mice as compared with Sparc ${ }^{+/+}$counterparts. (D) The incidence of upper tract urothelial tumors in the 15- to 25-week and greater than 25-week cohorts. ${ }^{*} P<0.05$; Fisher exact test. (E) The incidence of metastasis in Sparc $^{+/+}$and Sparc ${ }^{-/-}$mice in 15- to 25-week and greater than 25-week cohorts ${ }^{*} P<0.05$; Fisher exact test. (F) Incidence and number of visible lung metastases in the 15- to 25 -week and greater than 25 -week cohorts. ${ }^{*} P<0.05 ; \chi^{2}$ test; ${ }^{\star \star} P<0.05$, Student's $t$ test. (G) Representative H\&E-stained lung sections showing larger size of lung metastases in Sparc ${ }^{-/-}$lungs. Original magnification, $\times 100$. Only animals with invasive primary tumors were considered for analyses in $\mathbf{D}-\mathbf{G}$. 


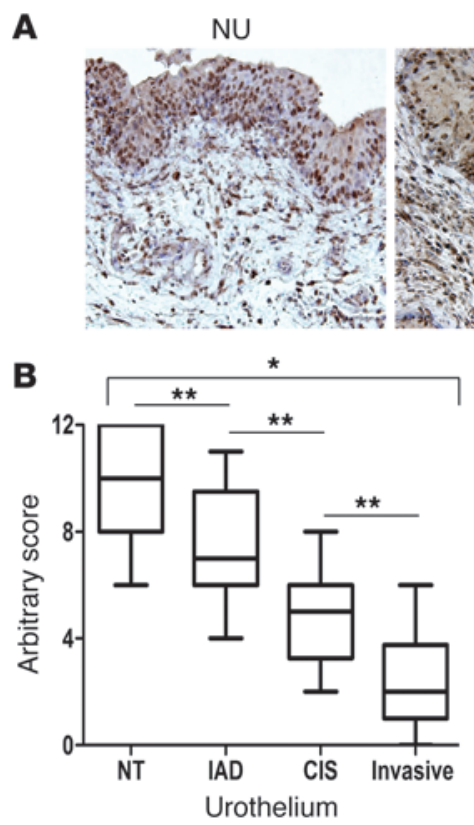

IAD

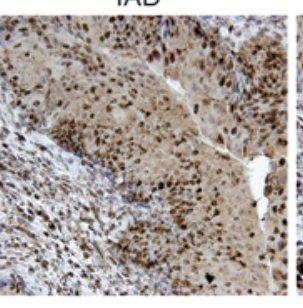

CIS

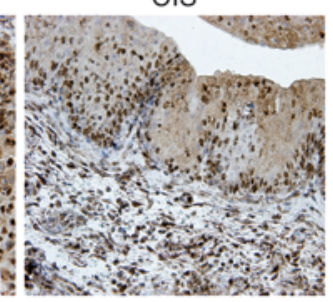

Invasive

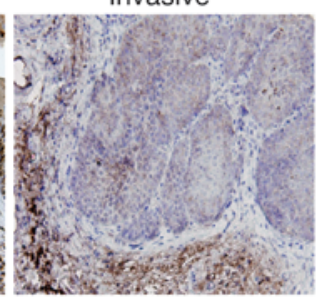

C

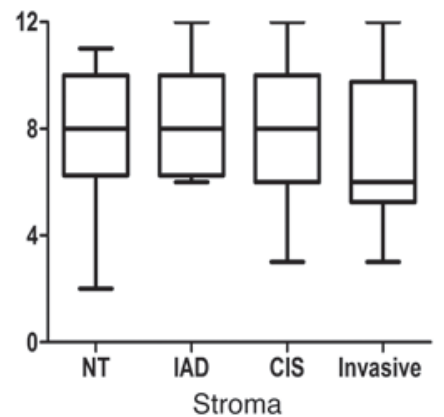

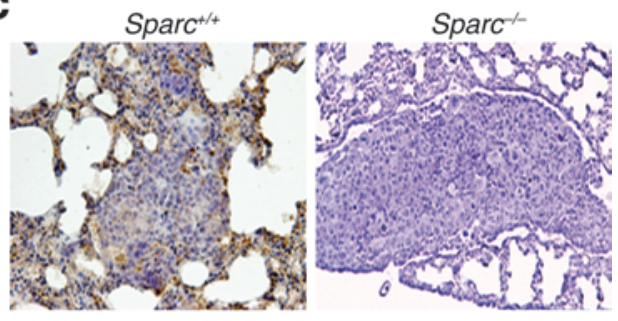

Figure 3

IHC expression of SPARC in bladders of Sparc ${ }^{+/+}$mice during multistep carcinogenesis. (A) Immunostaining of sections of dissected bladder lesions showing the differential expression of SPARC in the urothelium and lamina propria. Original magnification, $\times 200$. (B) The 2 arbitrary scores for intensity and frequency of SPARC immunostaining in the indicated groups of urothelium (left) and stromal (right) compartments were transformed into CESs as described in Methods. Boxes represent the mean \pm SEM, with whiskers minimum and maximum scores for each group ( $n=15$ animals/ group). *Correlation of composite scores in association with tumor grades by Kruskal-Wallis 1-way ANOVA followed by Dunn's multiple comparison post-hoc test. ${ }^{* *}$ Correlation of composite scores between indicated groups by 1-way ANOVA with Bonferroni's multiple comparison post-hoc test. (C) Immunostaining of SPARC protein in representative lung nodules from Figure 2G. Original magnification, $\times 200$.

disease progressed (Figure 3A). In normal bladder, SPARC expression was cytoplasmic and nuclear in all urothelial layers and in stromal cell types. With urothelial transformation, nuclear and cytoplasmic SPARC expression in cancerous urothelium diminished as a function of disease progression (Figure 3B). Interestingly, the expression of SPARC in $\mathrm{Sparc}^{+/+}$mice with lung metastases was observed exclusively in the surrounding stromal cells, not in cancer cells (Figure 3C).

Bladders of Sparc ${ }^{-/-}$mice show enhanced accumulation of ROS. There is a causal link between oxidative stress and cancer, where ROS produced by transformed epithelial cells and activated stromal and inflammatory cells account for DNA damage and genomic instability $(3,6,31,32)$ and further contribute to carcinogenesis. Hence, we assessed accumulation of superoxide anions by staining freshly frozen bladder sections with dihydroethidine (DHE), whose oxidation gives rise to the fluorescent derivative ethidene $(31,33)$. We found enhanced ROS accumulation was associated with progression of urothelial pathology in $\mathrm{Sparc}^{-1-}$ mice more than in matched Sparc ${ }^{+/+}$counterparts (Figure 4A). We also detected increased 8-OHdG, suggestive of oxidative- and inflammationmediated DNA damage, in bladders of BBN-treated Sparc ${ }^{-1}$ mice compared with the Sparc ${ }^{+/+}$(Figure 4B). Because ROS results in lipid peroxidation and oxidation of proteins that contribute to carcinogenesis via activation of oncogenic transcription factors such as NF-кB and AP-1 $(34,35)$, we determined the levels of lipid peroxidation product 8 -isoprostane and found higher levels in Sparc ${ }^{-1}$ mice compared with their wild-type counterparts (Figure 4C). Moreover, markers of protein oxidation, sulfiredoxin, and nicotinamide $\mathrm{N}$-methyl transferase (NNMT) were significantly increased in bladder tumor tissues of Sparc ${ }^{-1}$ mice (Figure 4D). Both have been correlated with progression and invasiveness of many cancers, including bladder $(34,36)$.

Loss of SPARC has an impact on cell-cycle regulators and inflammatory protein expression. Next we sought to determine whether the pathologic lesions found in Sparc ${ }^{-1-}$ mice were associated with a greater degree of cell-cycle dysregulation. As shown in Figure 5A, early phases of urothelial preneoplasia and neoplasia are associated with increased cell proliferation in Sparc ${ }^{-1-}$ mice, as evidenced by Ki67 immunostaining, which was almost exclusive in the preneoplastic and neoplastic urothelia and not in other cells in the underlying stroma. Therefore, we dissected urothelia of bladders from both genotypes and determined the differential expression of cellcycle regulatory proteins in sequential stages of disease. We found that dissected urothelia from Sparc S $^{-/}$mice exhibited increased expression of cell-cycle progression proteins and cyclin D1, A1, and E2 concomitant with decreased expression of their inhibitory proteins p21 and p27 (Figure 5B and Supplemental Figure 1; supplemental material available online with this article; doi:10.1172/ JCI64782DS1). Next we isolated primary normal urothelial (NU) and cancerous (UC) urothelial cells (Supplemental Figure 2) and found that Sparc ${ }^{-1-}$ UC cells exhibited 5-fold higher proliferation rates than their Sparc ${ }^{+/+}$counterparts (Figure 5B), whereas NU cells form Sparc ${ }^{-1-}$ mice exhibited only 50\% faster proliferation rate than their Sparc ${ }^{+/+}$counterparts. The enhanced proliferation rate in UC cells was accompanied by increased ROS generation as determined 
A
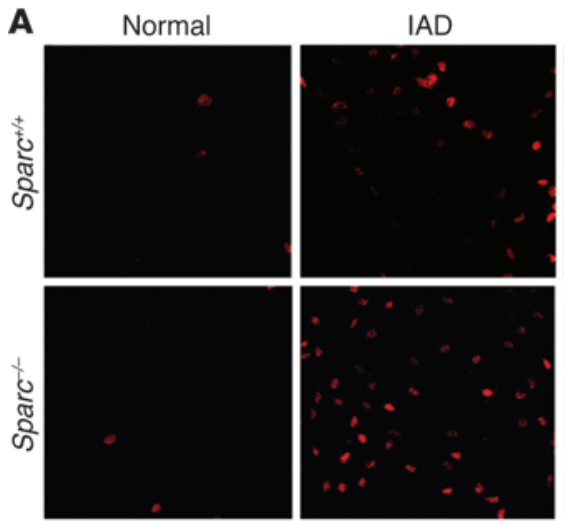

B
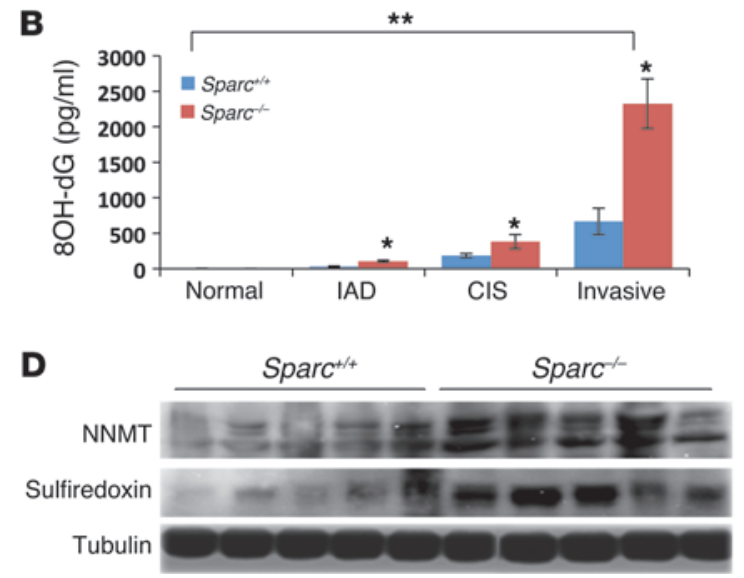

CIS

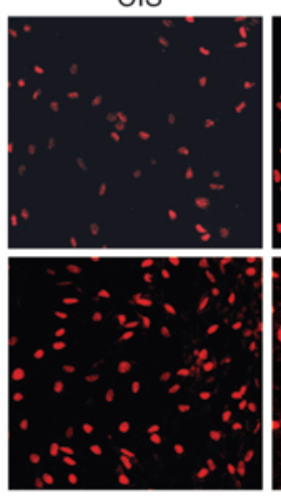

Invasive

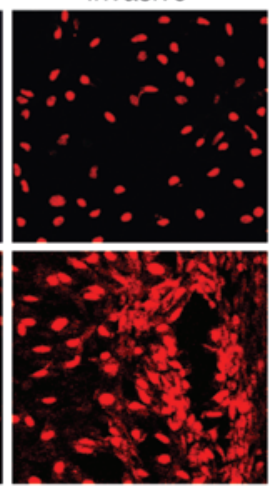

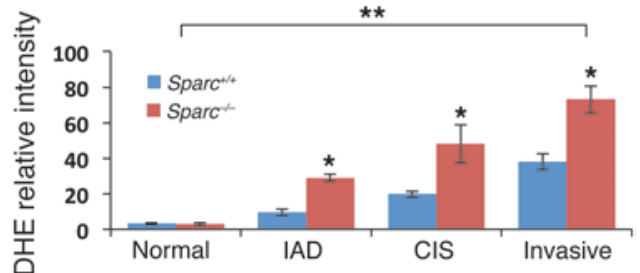

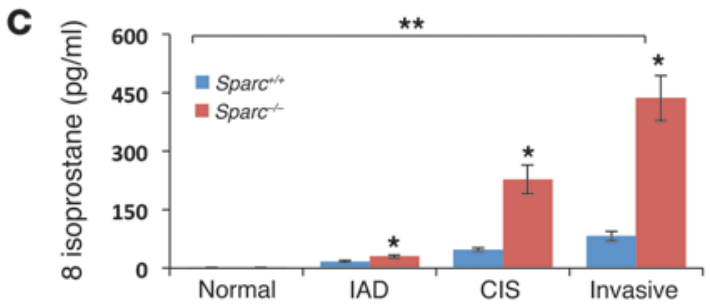

\section{Figure 4}

Lack of SPARC is associated with augmented ROS accumulation, DNA damage, lipid peroxidation, and protein oxidation. (A) Representative bladder cryosections from Sparc ${ }^{-/}$and Sparc ${ }^{+/+}$mice were incubated with $10 \mu \mathrm{M}$ DHE for 15 minutes at $37^{\circ} \mathrm{C}$. Cells staining positively for the oxidized dye were identified by fluorescent microscopy (DHE fluorescence was detected with excitation/emission at $518 / 605 \mathrm{~nm}$ ) and quantified by ImageJ analysis software. Original magnification, $\times 200$. Bars represent mean \pm SEM $\left(n=4\right.$ animals/cohort, 10 sections each). ${ }^{*} P<0.05$ between Sparc ${ }^{-/}$and Sparc ${ }^{+/}$, Student's $t$ test; ${ }^{* \star} P<0.05$ between different cohorts, 1-way ANOVA. (B) DNA damage in total DNA extracted from dissected bladders was determined by $8-O H d G$ EIA per manufacturer's instructions. (C) Lipid peroxidation was determined in bladder lysates by 8-isoprostane EIA per manufacturer's instructions. ${ }^{*} P<0.05$ between Sparc ${ }^{-/}$and Sparc ${ }^{+/+}$, unpaired 2-tailed Student's $t$ test; ** $P<0.05$ between different cohorts, 1-way ANOVA. (D) Western blots of bladder lysates of Sparc ${ }^{-/}$and Sparc Sp $^{+/+}(25 \mu \mathrm{g}, n=5)$ showing increased levels of protein oxidation markers NNMT and sulfiredoxin. Equal protein loading was confirmed by reprobing blots with tubulin.

by increased intracellular $\mathrm{H}_{2} \mathrm{O}_{2}$ production by measuring the fluorescence intensity of dichlorodihydrofluorescein (DCF). Interestingly, enhanced $\mathrm{H}_{2} \mathrm{O}_{2}$ production in $\mathrm{Sparc}^{-/-}$cells was partially inhibited by adding exogenous SPARC (10-20 $\mu \mathrm{g} / \mathrm{ml})$ but not the anti-oxidant $\mathrm{N}$-acetyl cysteine (NAC) (Figure 5D), which only inhibited $\mathrm{H}_{2} \mathrm{O}_{2}$ from normal and cancerous cells without affecting their proliferation.

ROS were also shown to be major contributors to cancer development through activation and phosphorylation of p38 MAPK and JNK, with subsequent activation of multiple signaling pathways that converge into activation of NF- $\mathrm{KB}$ and AP-1, which are the major transcription factors orchestrating cancer progression. NF-кB and AP-1 $(34,35,37)$. Higher levels of ROS in Sparc ${ }^{--}$urothelia prompted us to evaluate potential downstream signaling pathways. In addition, SPARC has been shown to suppress the activation of NF- $\mathrm{KB}$ in ovarian cancer cells in response to inflammatory cells and mediators $(7,11)$. Western blot analysis of bladder lysates revealed marked increase in the phosphorylation of p38 MAPK,
JNK, and c-Jun as well as p65-NF-кB in Sparc ${ }^{-1-}$ tissue lysates of early pathological lesions compared with their $\mathrm{Sparc}^{+/+}$counterparts (Figure 6A and Supplemental Figure 3A). Immunostaining of bladder tissue from Sparc ${ }^{+/+}$and $\mathrm{Sparc}^{-/-}$revealed a progressive increase in the phosphorylation and nuclear localization of c-Jun and p65-NF-кB (Figure 6, B and C, and Supplemental Figure 3, B and $C$, respectively) in malignant $S$ parc ${ }^{-/}$urothelia and adjacent stroma compared with that in Sparc ${ }^{+/+}$counterparts. Earlier reports indicated the upregulation of proinflammatory cytokines in orthotopic ovarian, prostate, and pancreatic tumors growing in Sparc-mice $(7,8,11)$. In addition, SPARC has been shown to antagonize the effects of proinflammatory, proangiogenic cytokines and mediators (summarized in refs. 7,11). Therefore, we determined the levels of NF- $\kappa$ B- and AP-1-dependent cytokines IL-6, CCL2, VEGF, and TNF- $\alpha$ and found that they significantly increased as a function of disease progression and were significantly increased in the absence of SPARC (Supplemental Figure 4A). In addition, the levels of CCL3/macrophage inflammatory protein $1 \alpha$ (MIP1 $\alpha)$, CXCL2 
A
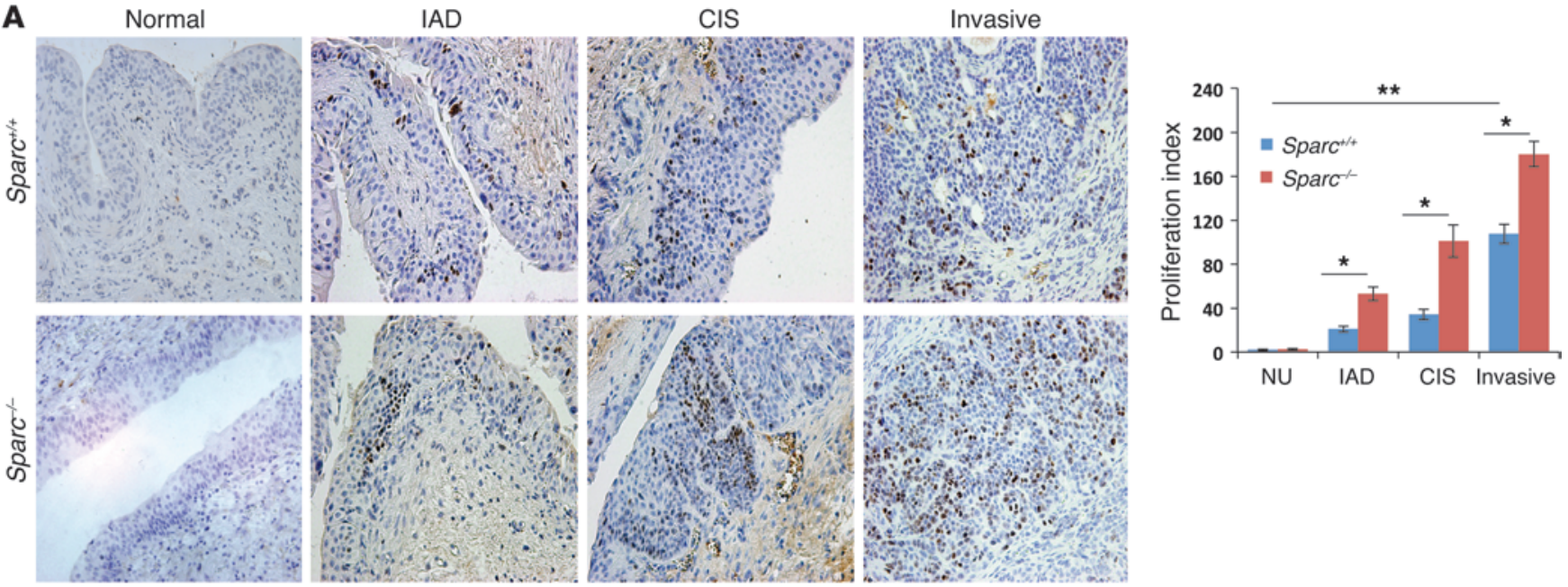

B

NU

IAD

$\mathrm{CIS}$

Invasive
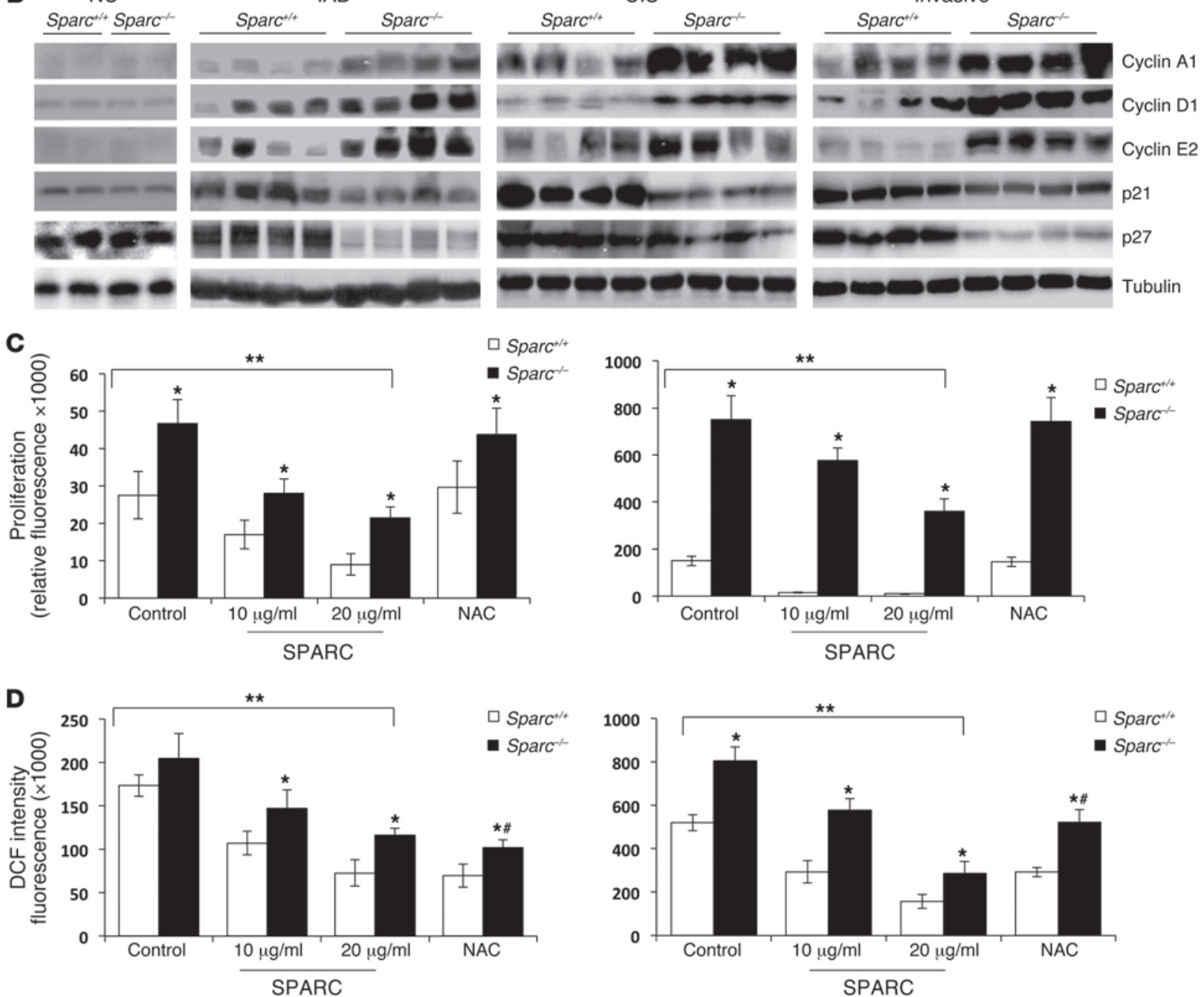


\begin{abstract}
Figure 5
Loss of SPARC enhances BBN-induced cell-cycle dysregulation in urothelium and in murine urothelial cells in vitro. (A) Photomicrographs of Ki67 immunostaining in dissected bladder tissues from the indicated cohorts. Original magnification, $\times 200$. Bars represent mean \pm SEM of Ki67-positive nuclei counted in 6 random HPF/slide $(n=5$ animals of each genotype/group). ${ }^{\star} P<0.05$ comparing Sparc ${ }^{-/-}$and Sparc ${ }^{+/+}$, 2-tailed Student's $t$ test. ${ }^{* *} P<0.05,1$-way ANOVA. (B) Western blot analysis of $25 \mu \mathrm{g}$ protein of dissected Sparc ${ }^{+/+}$and Sparc $^{-/-}$urothelium from bladders with the indicated pathology for cell-cycle proteins and their inhibitors. Protein loading was verified by probing the membranes with tubulin. Quantification can be found in Supplemental Figure 1. (C)

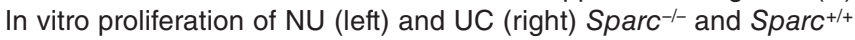
urothelial cells was determined by CyQuant assay. Cells were allowed to attach in 96 wells for 6 hours in CGM, then were switched to medium with $2 \%$ FBS (basal) in the presence and absence of $0-20 \mu \mathrm{g} / \mathrm{ml}$ SPARC or $1 \mathrm{mM}$ NAC for 48 hours. (D) Generation of $\mathrm{H}_{2} \mathrm{O}_{2}$ was determined by measuring the fluorescent intensity of DCF of Sparc ${ }^{-/}$and Sparc $^{+/+}$murine urothelial cells (NU, left; UC, right) under indicated experimental conditions (excitation/emission 488/520 nm). Bars represent mean \pm SEM of 2 independent experiments performed in quadruplicate. ${ }^{*} P<0.05$ comparing Sparc ${ }^{-/-}$and $S p a r c^{+/+}$, unpaired 2-tailed Student's $t$ test; ${ }^{* *} P<0.05$ comparing the doses of exogenous SPARC, 1-way ANOVA; ${ }^{~} P<0.05$ comparing treatment with NAC to control/ carrier conditions.
\end{abstract}

(MIP2 $\alpha$, Gro- $\beta$ ), CSF, and monocyte-CSF (M-CSF) were also significantly elevated, with highest levels observed in invasive disease

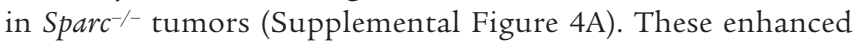
cytokine/chemokine levels were associated with greater infiltration by tumor-associated macrophages (TAM) as determined by mac1-positive immunostaining (Supplemental Figure 4B). These findings indicate that in the absence of SPARC, accelerated inflammation accompanies urothelial preneoplasia as a consequence of substantial activation of NF- $\mathrm{KB}$ and AP-1 transcription factors in both urothelium and stroma, with progressive increase in downstream target chemokines/growth factors.

Loss of SPARC in TAMs enhances their inflammatory phenotype. Having shown that TAMs are increased in $\mathrm{Sparc}^{-/-}$bladder lesions compared with Sparc ${ }^{+/+}$(Supplemental Figure 4A), we sought to determine the contribution of macrophage-SPARC to bladder cancer progression. We isolated monocytes from Sparc ${ }^{-/-}$and Sparc ${ }^{+/+}$ bone marrows and induced their differentiation into macrophages as described (38). Under basal nonstimulated conditions, we did not observe any difference in the proliferation rates between Sparc ${ }^{+/}$and Sparc ${ }^{-/}$macrophages (Supplemental Figure 5A). Neither exogenous SPARC nor genetic manipulation of SPARC expression by overexpression or knockdown affected their proliferation (Supplemental Figure 5A). Sparc ${ }^{+/+}$macrophages exhibited transient increase in SPARC protein expression that peaked after 3 days, then declined to basal level at day 7 after stimulation (Supplemental Figure 5B). In addition, under basal unstimulated conditions, no differences in the activity of AP- 1 and NF- $\kappa$ B promoters between $\mathrm{Sparc}^{+/+}$and Sparc ${ }^{-/-}$macrophages were detected, as determined by luciferase reporter assays (Supplemental Figure 5, C and D). Coincubation of primary Sparc ${ }^{+/+}$and Sparc $-/$UC cells with $\mathrm{Sparc}^{+/+}$and $\mathrm{Sparc}^{-/-}$macrophages increased AP-1 and NF-KB (Figure 7, A and B) promoter activity in UC cells and macrophages. Under all conditions, Sparc ${ }^{-/}$UC cells and macrophages exhibited substantially higher AP-1 and NF-кB promoter expression compared with Sparc ${ }^{+/}$, with the highest levels occurring when Sparc -/ macrophages and UC cells were coincubated.
We next investigated the effect of macrophage-SPARC on the interaction of macrophages with tumor cells. Surprisingly, $\mathrm{Sparc}^{+/+}$and Sparc ${ }^{-/}$macrophages migrated similarly toward complete growth medium (CGM) (Figure 7C); however, Sparc ${ }^{-1-}$ macrophages exhibited significantly more migration toward conditioned medium (CM) from UC cells, with maximum migration observed when CM from Sparc ${ }^{-1-} \mathrm{UC}$ cells was used as an attractant (Figure 7C). Furthermore, CM of Sparc ${ }^{-/-}$macrophages induced significantly more invasiveness of UC cells than CM from Sparc ${ }^{+/+}$ macrophages, with maximum invasiveness exhibited by Sparc ${ }^{-1-}$ UC cells toward CM of Sparc ${ }^{-1-}$ macrophages (Figure 7D). Furthermore, stimulation of Sparc ${ }^{+/+}$and $S_{\text {parc }}{ }^{-/}$macrophages with $\mathrm{CM}$ of Sparc ${ }^{+/+}$and Sparc ${ }^{-1}$ UCs increased the production of $\mathrm{H}_{2} \mathrm{O}_{2}$ from macrophages as determined by DCF (Supplemental Figure 5E). When Sparc ${ }^{+/+}$and Sparc ${ }^{-/-}$macrophages were stimulated with $\mathrm{H}_{2} \mathrm{O}_{2}$, which is the prototypical ROS encountered in many tumor microenvironments including that of bladder cancer, the production of inflammatory cytokines by macrophages of either genotype was significantly increased over the nonstimulated controls (Supplemental Figure 5F). Moreover, $\mathrm{H}_{2} \mathrm{O}_{2}$-induced cytokine production by Sparc ${ }^{-1}$ macrophages was markedly higher than Sparc ${ }^{+/+}$ counterparts. These results indicate that increased SPARC expression by Sparc ${ }^{+/+}$macrophages during their differentiation attenuates their proinvasive inflammatory characteristics.

Loss of SPARC in cancer-associated fibroblasts enhances their inflammatory phenotype. To determine the contribution of SPARC on stromal fibroblasts in the context of BBN-induced carcinogenesis and progression, we isolated primary stromal fibroblasts from cancerous (cancer-associated fibroblasts [CAF]) and noncancerous (normal fibroblasts [NF]) Sparc ${ }^{-1-}$ and Sparc ${ }^{+/+}$bladders (ref. 39 and Supplemental Figure 3A). Consistent with earlier reports $(38,40-42)$, under basal conditions, Sparc ${ }^{-1-}$ NF exhibited 3.2-fold more proliferation, whereas Sparc ${ }^{-1-}$ CAFs exhibited 0.9-fold more proliferation than Sparc ${ }^{+/+}$counterparts: an effect that was reversed by exogenous SPARC $(20 \mu \mathrm{g} / \mathrm{ml}$; Supplemental Figure 6A). Next, we determined the expression of SPARC protein in $\mathrm{Sparc}^{+/+} \mathrm{NF}$ during their differentiation in response to $\mathrm{H}_{2} \mathrm{O}_{2}$ and CM from 72-hour murine bladder cancer cells (MB49). We also tested the effects of 8-isoprostane, another marker of oxidative stress/damage that progressively increased during preneoplasia and neoplasia (Figure 4C). We found SPARC increased, peaking at day 3 (Supplemental Figure 6B). By day 10, a significant increase in expression of IL-6, CCL2, VEGF and TNF- $\alpha$ transcripts over unstimulated $\mathrm{NF}$ was also detected. Similar stimulation of Sparc ${ }^{-1}$ NFs with $\mathrm{H}_{2} \mathrm{O}_{2}$ and CM resulted in levels of cytokines higher than those of Sparc $\mathrm{Pat}^{+/}$ counterparts (Supplemental Figure 6C). Notably, at all time points during differentiation, NFs were not proliferating, with $70 \%-85 \%$ cells in $\mathrm{G}_{1}$ phase and less than $0.17 \%$ apoptotic cells, as determined by propidium iodide staining and flow cytometry.

$\mathrm{Sparc}^{+/+}$NF exhibited 2.4-fold more migration toward CGM vs. their Sparc ${ }^{-/}$counterparts; whereas Sparc ${ }^{+/+}$CAFs exhibited enhanced migration toward growth medium, Sparc ${ }^{+/+} \mathrm{UC}$, and $\mathrm{Sparc}^{-/-}$ CM (1.6-, 1.8-, and 2-fold increase over the Sparc-/-, respectively). Given that CAFs associated with developing neoplasia exhibit a proinflammatory signature driven by NF- $\mathrm{BB}$ and AP-1 (summarized in ref. 43), we investigated the effect of SPARC on the activity of NF- $\kappa \mathrm{B}$ and AP-1 promoters in normal and cancerous fibroblasts and urothelial cells. Under basal unstimulated conditions, there was no significant difference in AP-1 and NF- $\mathrm{BB}$ promoter activity between normal fibroblasts and NU cells of either genotype, whereas Sparc /- UC cells exhibited significantly higher activity than Sparc ${ }^{+/}$ 
A

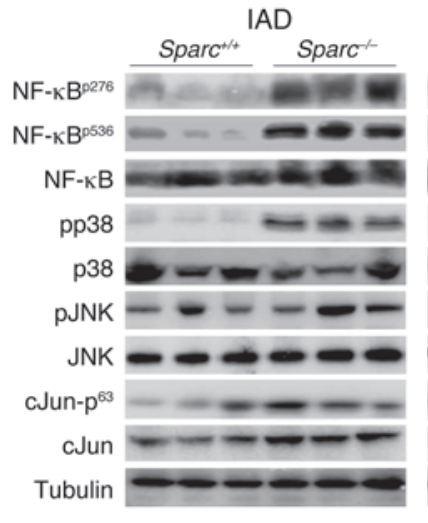

B

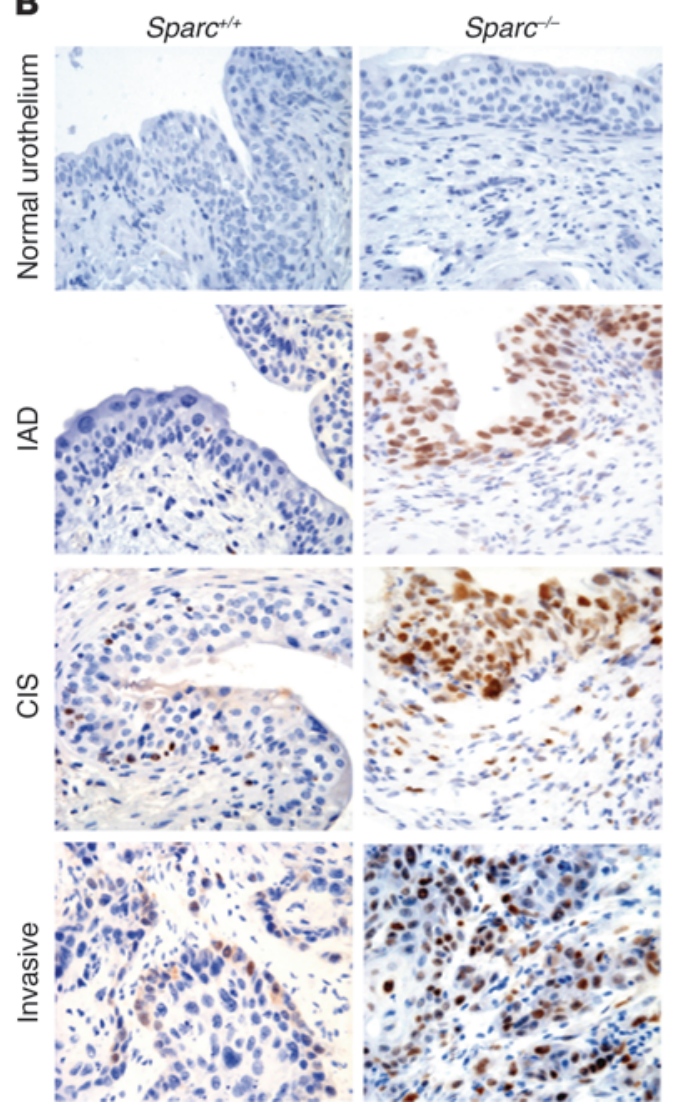

CIS

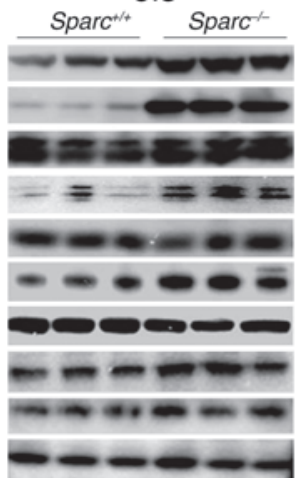

C

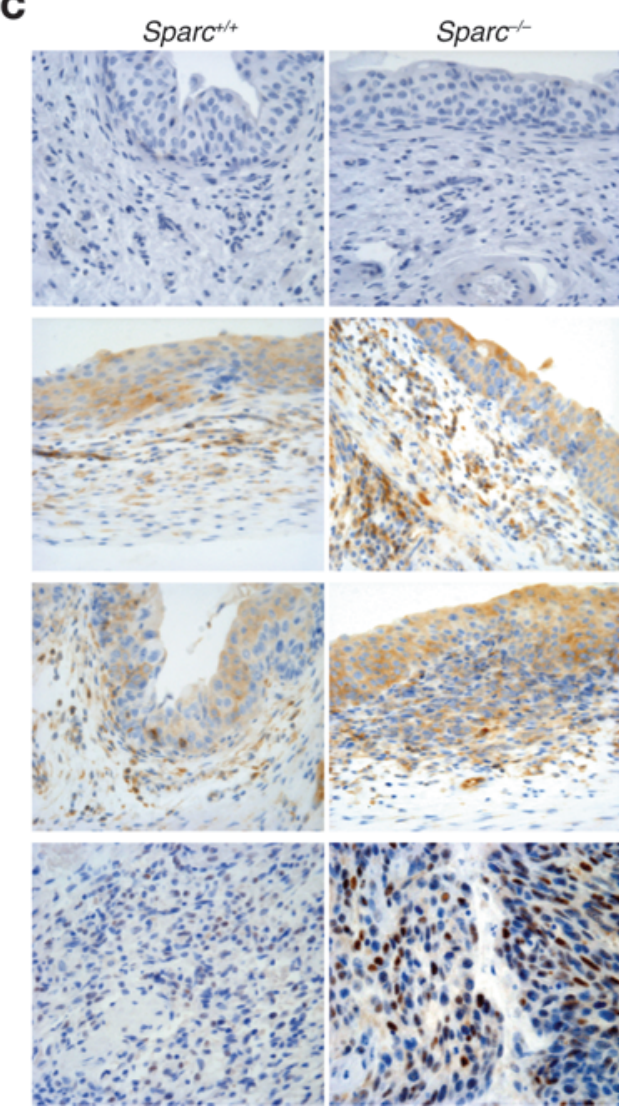

\section{Figure 6}

Bladder carcinogenesis is associated with increased activation of NF- $\mathrm{KB}$ and p38-JNK-AP-1. (A) Western blot analysis of lysates from dissected bladder tissue from BBN-treated Sparc ${ }^{-/}$and Sparc ${ }^{+/+}$mice with preneoplasia (IAD and CIS; $n=3$ each) showing increased phosphorylation of NF-кB p65 (Ser276 and Ser536), p38, SAPK/JNK (Thr183/Tyr185), and c-Jun (p63). Equal protein loading was confirmed by tubulin. (B and C) Immunostaining of bladder tissue sections shows the nuclear localization of phosphorylated c-Jun and cytoplasmic and nuclear localization and scoring of p65-NF-кB, respectively, in the normal, IAD, CIS, and invasive cancerous lesions. Original magnification, $\times 200$.

UC cells (Figure 8, A and B). Coincubation of primary Sparc ${ }^{+/+}$and Sparc/- UC cells with Sparc ${ }^{\prime /}$ and Sparc $/-$ CAFs for 24 hours resulted in marked increase in AP-1 and NF-kB promoter activation in UC cells and CAFs. Under all the experimental conditions, Sparc-1- UC cells and CAFs exhibited significantly higher activity of AP- 1 and NF-KB promoters compared with Sparc ${ }^{+/}$, with the highest activation occurring when Sparc ${ }^{-}$CAFs and UC cells were coincubated (Figure 8, C and D). Furthermore, coincubation of Sparc ${ }^{+/+}$and
Sparc ${ }^{-1}$ CAFs and UC cells revealed that Sparc $/-\mathrm{CAFs}$ were more potent inducers of UC cell invasiveness than $\mathrm{Sparc}^{++} \mathrm{CAFs}$ (Figure 8E). In either genotype, CAFs secreted higher levels of IL-6, CCL2, TNF- $\alpha$, and VEGF in their CM than NF. Interestingly, the production of TGF- $\beta$ and SDF1/CXCL12 was primarily secreted by fibroblasts in vitro, suggesting they are the major contributors of these factors. The inflammatory secretome of Sparc/- CAFs was higher than that of Sparc ${ }^{++}$(Supplemental Figure 6, D and E). 

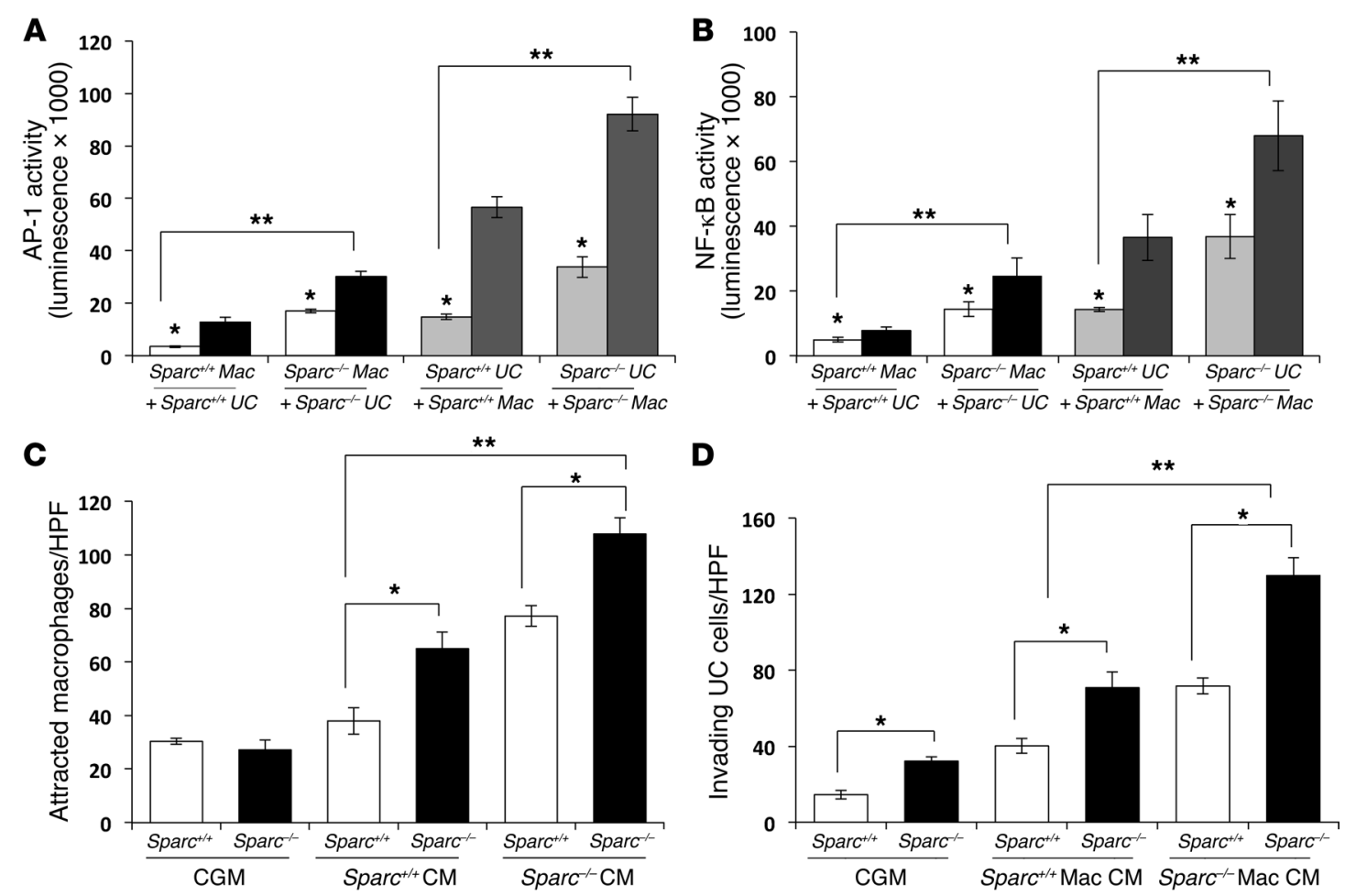

Figure 7

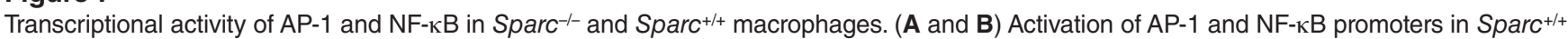
and Sparc ${ }^{--}$macrophages (Mac) cocultured with UC cells for 24 hours. Bars represent mean \pm SEM of 2 independent experiments performed in triplicate. ${ }^{*} P<0.05$, comparing Sparc ${ }^{+/+}$UCs cultured with Sparc ${ }^{+/+}$and Sparc ${ }^{-/-}$Macs; ${ }^{* *} P<0.05$, comparing Sparc ${ }^{-/-}$UCs cultured with Sparc ${ }^{+/+}$ and Sparc ${ }^{-/}$Macs; ${ }^{* * *} P<0.05$, comparing Sparc ${ }^{-/-}$and Sparc ${ }^{+/+}$UCs cultured with Sparc ${ }^{+/+}$and Sparc ${ }^{-/}$Macs. (C) Migration of primary Macs toward CGM, CM of Sparc ${ }^{+/+}$and Sparc ${ }^{-/-}$UC cells. Bars represent mean \pm SEM of 2 independent experiments performed in quadruplicate. ${ }^{*} P<0.05$ between migration of Sparc ${ }^{-/-}$and Sparc ${ }^{+/+}$Macs toward each attractant; ${ }^{\star \star} P<0.05$ between Sparc ${ }^{-/-}$and Sparc ${ }^{+/+}$Macs migrating toward Sparc ${ }^{+/+}$ and Sparc ${ }^{-/}$CM. (D) CM from Sparc ${ }^{-/}$and Sparc ${ }^{+/+}$Macs to stimulated Sparc ${ }^{-/}$and Sparc S $^{++}$UC cells Matrigel-invasiveness in Transwell assays. Bars represent mean \pm SEM $(n=4) .{ }^{*} P<0.05$ between Sparc ${ }^{-/}$and Sparc ${ }^{+/+}$UC cells migration toward $\mathrm{CM}$; ${ }^{* *} P<0.05$ between the effect of $\mathrm{CM}$ of Sparc ${ }^{-/}$and Sparc ${ }^{+/+}$Macs on UCs invasiveness. All comparisons were performed with unpaired 2-tailed Student's $t$ test.

Finally, we determined whether SPARC exerts an antiangiogenic effect and found that Sparc ${ }^{-1-}$ preneoplastic and neoplastic bladders exhibited enhanced vascularity compared with those in $\mathrm{Sparc}^{+/+}$mice (Supplemental Figure 7, A-D).

Host SPARC suppresses murine bladder cancer growth and metastasis. Given that Sparc $\mathrm{Sumors}^{--}$were more metastatic and exhibited a significantly increased angiogenic and inflammatory phenotype, with higher levels of inflammatory cytokines/chemokines (Supplemental Figure 4A), we were prompted to determine chemokine levels in the lungs corresponding to the bladder tissues examined in Supplemental Figure 4A. Interestingly, we found a progressive increase in their levels in matching lung tissues as a function of disease progression with significantly higher levels in $\mathrm{Sparc}^{-/}$compared with $\mathrm{Sparc}^{+/+}$lungs (Supplemental Figure 8A). In addition, macrophage infiltration was significantly higher in $\mathrm{Sparc}^{-/-}$compared with the $\mathrm{Sparc}^{+/+}$lung metastases (Supplemental Figure 8B). These data are consistent with our recent reports that metastatic colonization of lung is instigated by bladder cancer cells and enhanced by reactive host macrophages preconditioning the metastatic niche $(28,29)$. Therefore, we sought to determine the contribution of cancer cell vs. stromal cell SPARC in bladder cancer metastasis by using MB49 cells $(28,29)$, a syngeneic model of spontaneous metastasis that expresses detectable levels of SPARC (Figure 9A).
Two weeks after s.c. injection, tumor volumes were greater, while actuarial survivals were shorter in Sparc ${ }^{-/-}$mice compared with their Sparc ${ }^{+/+}$counterparts (Figure 9, B and C). s.c. tumors growing in $\mathrm{Sparc}^{-1-}$ mice exhibited increased macrophage infiltration (Supplemental Figure 9A) and mean vascular density (Supplemental Figure 9B) vs. those growing in Sparc $^{+/+}$mice. In addition, all mice with s.c. tumors developed lung metastases, with metastases in Sparc ${ }^{-/}$mice being more infiltrated with macrophages (Supplemental Figure 9C). Consistent with our findings in the carcinogenesis model, the levels of inflammatory cytokines in matched s.c. tumors and lung metastases were higher in Sparc-/- mice (Supplemental Figure 9D). These results further support that host SPARC suppresses primary tumor growth and metastasis, exerting anti-angiogenic and antiinflammatory effects at each site.

To examine the possibility that increased metastases in Sparc ${ }^{-1}$ mice are due to faster growing and/or larger primary tumors and to determine the relevance of SPARC suppression on the latter phase of metastasis, namely metastatic colonization, we injected a fixed number of MB49 i.v. in Sparc ${ }^{-/}$and Sparc ${ }^{+/+}$mice expressing GFP and examined lung colonization at early time points. After perfusion of the dissected lungs to dislodge nonadherent cells, we found that as early as 24 hours after injection, the arrest and colonization of MB49-GFP cells was significantly higher in Sparc-- mice 

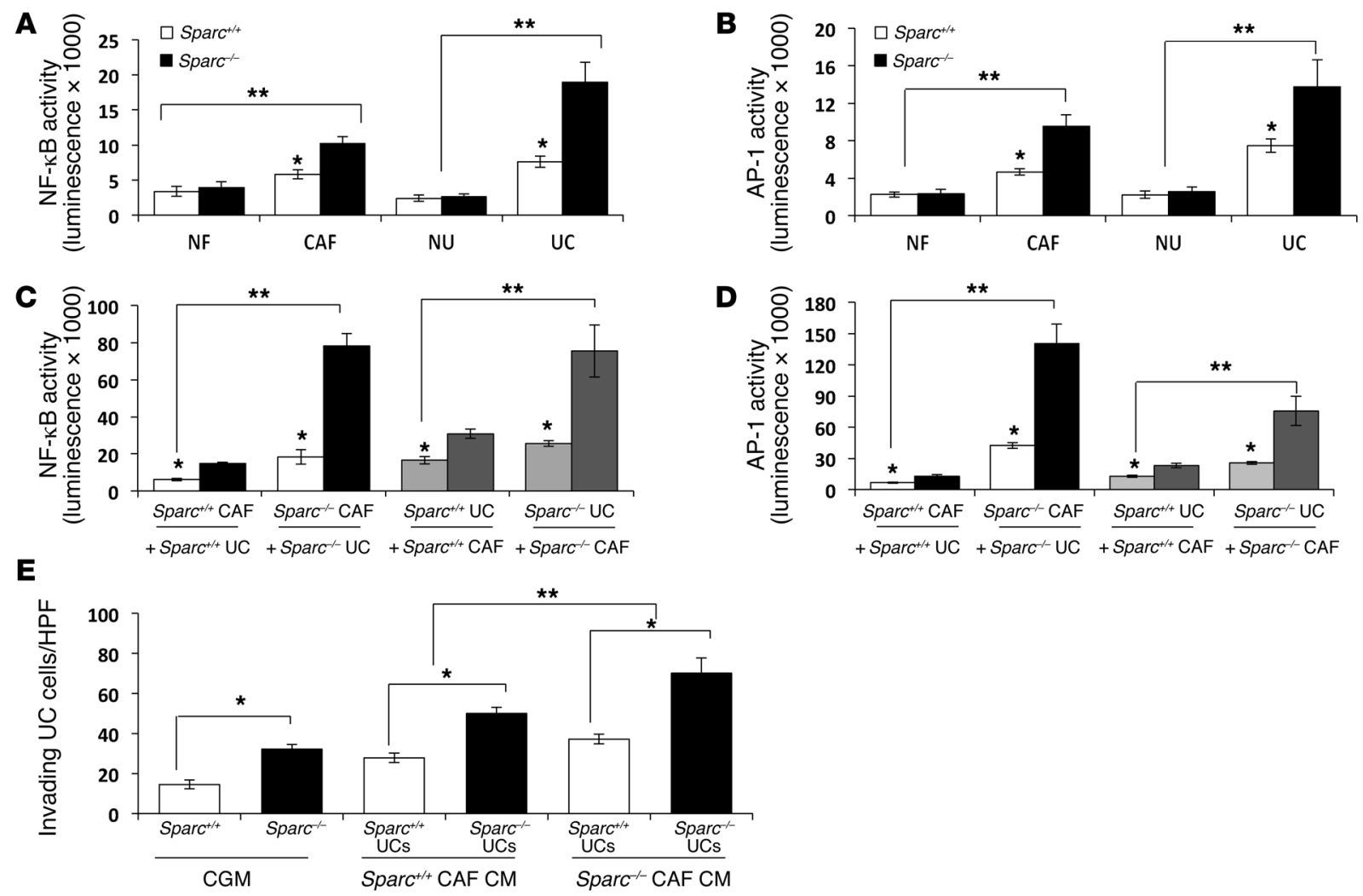

\section{Figure 8}

Reciprocal activation of AP-1 and NF-кB in Sparc ${ }^{-/}$and Sparc ${ }^{+/+}$urothelial cells and stromal fibroblasts. (A and B) The promoter activity of NF- $\mathrm{KB}$ and AP-1 was determined by measuring the luciferase activity in primary Sparc ${ }^{-/}$and Sparc ${ }^{+/+} \mathrm{NF}, \mathrm{CAF}, \mathrm{NU}$, and UC cells transfected with pAP-1-luc and pNF-кB-luc reporter plasmids or pGL2 control vector for 24 hours as described in Methods. (C and D) Activation of NF-kB

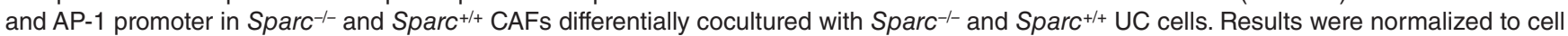
proliferation determined by CyQuant. Bars represent mean \pm SEM of 2 independent experiments performed in triplicate. ${ }^{*} P<0.05$. (E) The ability of Sparc ${ }^{-/}$and Sparc ${ }^{+/+}$CAFs to stimulate Sparc ${ }^{-/-}$and Sparc SPC $^{+/+}$cells was determined in Transwell invasion assays using CM of Sparc ${ }^{-/-}$and Sparc $^{+/+}$CAFs in the bottom chamber of Matrigel-coated filters and Sparc ${ }^{-/}$and Sparc ${ }^{+/+} \mathrm{UC}$ cells added onto the filters. Bars represent mean \pm SEM $(n=4) .{ }^{*} P<0.05$ between migration of Sparc ${ }^{-/}$and Sparc ${ }^{+/+}$UC cells toward CM; ${ }^{* *} P<0.05$ between the effect of CM of $S p a r c^{-/-}$and Sparc $^{+/+}$CAFs on UC invasiveness. All comparisons were performed with unpaired 2-tailed Student's $t$ test.

compared with Sparc ${ }^{+/+}$animals (Figure 9D). The number of fluorescent cells arrested/colonized in the lung progressively increased over 48 and 72 hours, with markedly more cells in Sparc ${ }^{-1}$ lungs. Together, these data indicate that host SPARC inhibits the early metastatic colonization in the lungs and that enhanced metastasis from the s.c. site is not exclusively due to faster growing or larger primary tumors in $\mathrm{Sparc}^{-1-}$ mice.

Tumor cell SPARC suppresses human urothelial carcinoma growth and lung colonization. Next, we sought to determine the relevance of SPARC in models of human bladder cancer by first determining the expression and effect of endogenous SPARC on human UC cells. We found that protein expression of SPARC is less in the tumorigenic T24T compared with poorly tumorigenic T24 isogenic line (27) and also found detectable SPARC expression in another tumorigenic UMUC3 cell line (Figure 10A). We then manipulated SPARC expression in UMUC3, T24, and T24T cell lines and found that forced expression of SPARC inhibited, while its depletion enhanced, cell proliferation in vitro (Figure 10A). Overexpression of SPARC in these cells was associated with decreased expression of cyclins A, D, and $\mathrm{E}$, concomitant with increased expression of their inhibitors p21 and p27, whereas depletion of SPARC in UMUC3 and T24 cells had an opposite effect (Figure 10B). Furthermore, in s.c. xenografts in nude mice, overexpression of SPARC reduced in vivo growth of UMUC3 and T24T cells, whereas its depletion enhanced growth and size of UMUC3 xenografts and increased tumorigenicity of T24 cells to a level similar to that of T24T (Figure 10C).

To determine the role of tumor-SPARC in lung colonization and extend the observed MB49 data in a human model, we used an experimental metastasis model $(28,29)$, injecting human bladder cancer cells genetically modified for SPARC expression into nude mice. We found that, in UMUC3 cells, forced expression of SPARC significantly inhibited the incidence and multiplicity of lung metastasis (Supplemental Figure 10A), whereas depletion of SPARC increased the number and size of lung metastases and was associated with deceased survival (Supplemental Figure 10B). Interestingly, depletion of SPARC in the nontumorigenic nonmetastatic T24 cells substantially increased their incidence and number of lung metastases comparable to their isogenic metastatic T24T cells. In contrast, expression of SPARC in T24T significantly abrogated their metastatic ability (Supplemental Figure 10B). To determine the effect of 
A
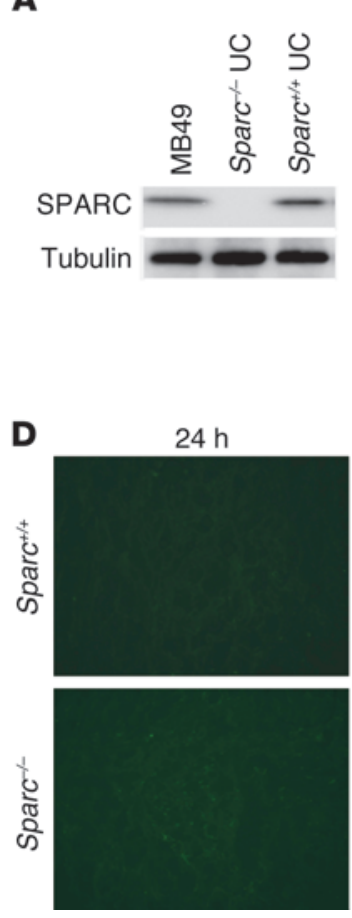

B

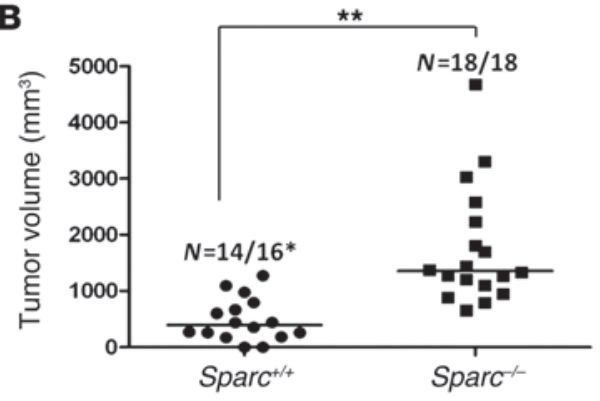

$48 \mathrm{~h}$
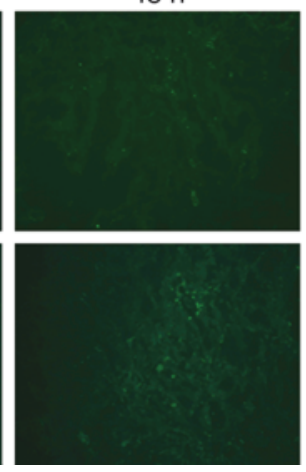

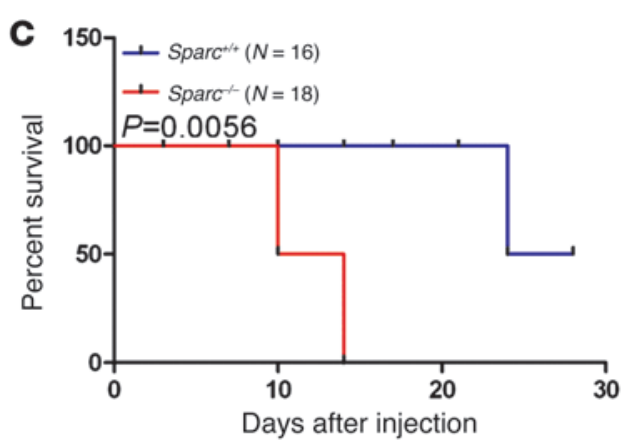

Figure 9

Host SPARC exerts a metastasis suppressor effect in murine models of bladder cancer. (A) Western blots of SPARC in MB49 (25 $\mu \mathrm{g})$ relative to primary Sparc ${ }^{+/+}$and Sparc ${ }^{-/}$UC cells. Equal protein loading was confirmed by tubulin. (B) Scatter plots of tumor take and volume of MB49 cells injected s.c. in Sparc ${ }^{-/}$and Sparc ${ }^{+/+}$mice. ${ }^{\star} P<0.05, \chi^{2}$ test; ${ }^{\star \star} P<0.05$, Student's $t$ test. (C) Kaplan-Meier curves showing the survival of Sparc $^{+/+}$and Sparc ${ }^{-/-}$mice after s.c. inoculation of MB49 cells. (D) Photomicrographs of GFP-MB49 cells i.v. injected in Sparc ${ }^{-/-}$and Sparc ${ }^{+/+}$mice at indicated time points. Bars represent mean \pm SEM of fluorescent intensity measured as described in Methods. ${ }^{*} P<0.05$, unpaired 2 -tailed Student's $t$ test, compared with Sparc ${ }^{+/+}$.

endogenous SPARC on early metastatic colonization, we injected UMUC3 cells harboring non-targeting shRNA (UMUC3-NTsh) and UMUC3 depleted of SPARC (UMUC3-shSP) labeled with green fluorescent tracker CMFDA and quantified fluorescent signal in the lungs after perfusion. We found UMUC3-shSP exhibited a dramatic increase in lung colonization as early as 24 hours after injection, and this was maintained for 48 and 72 hours as compared with control cells (UMUC3-NTsh; Supplemental Figure 10C). Collectively, our results indicate that endogenous SPARC exerts a metastasissuppressor effect on bladder cancer cells in part through inhibiting colonization in the lung early in the metastatic cascade.

\section{Discussion}

To clarify apparently conflicting reports regarding the role of SPARC in tumor formation and progression, we used a welldefined bladder carcinogenesis model $(3,30)$ in Sparc $^{-/-}$and $\mathrm{Sparc}^{+/+}$mice coupled with complementary murine and human models of disease $(28,29)$ as well as patient tumors to construct a comprehensive portrait of SPARC function in human bladder cancer. This approach is the first, to our knowledge, to clearly distinguish the role of tumor versus host SPARC.

Carcinogen exposure is known to be associated with progressive generation of ROS and markers of DNA, protein, and lipid oxidative damage as well as inflammatory cytokines/chemokines (5, $31,44)$. We show that in the absence of SPARC, cell-cycle deregulation and the initiation of urothelial atypia/dysplasia (Figures
2 and 3) are accelerated concomitant with increased production of ROS (Figure 4), driving oxidative damage and inflammation reciprocated by urothelia and stroma. The antiproliferative and cell-cycle inhibitory effects of SPARC in preneoplastic and neoplastic urothelia were due to cell-cycle arrest at the $G_{1} / S$ phase with downregulation of cyclins $\mathrm{A}, \mathrm{D}$, and $\mathrm{E}$ and upregulation of their inhibitors $\mathrm{p} 21$ and $\mathrm{p} 27$. Interestingly the production of ROS by Sparc ${ }^{-1-}$ urothelial cells in vitro was reversed by addition of SPARC, which inhibited cell proliferation and ROS generation and inflammation supporting a role for SPARC in restraining cellcycle progression and tumor cell proliferation. We also show that Sparc ${ }^{-/-}$mice increased accumulation of markers of oxidative damage, including nicotinamide NNMT and sulfiredoxin (Figure 4). Both are endogenous antioxidants that are involved in biotransformation of xenobiotics and morbidities characterized by oxidative stress (45). However, their overexpression has been observed in many invasive and metastatic tumors (summarized in ref. 46), including bladder, skin, and lung cancers $(34,36)$. In addition, they act as signaling molecules leading to progressive activation of p38 MAPK and stress-activated protein kinase (SAPK)/JNK. These latter two converge to activate AP-1 and NF- $\mathrm{BB}$, major orchestrators of inflammation, carcinogenesis, invasiveness, and metastasis $(31,44,47-49)$.

The role of tumor- versus host-SPARC cannot be distinguished by gene expression profiling, as the high expression of SPARC in the stromal compartment masks its downregulation/loss in the 
A

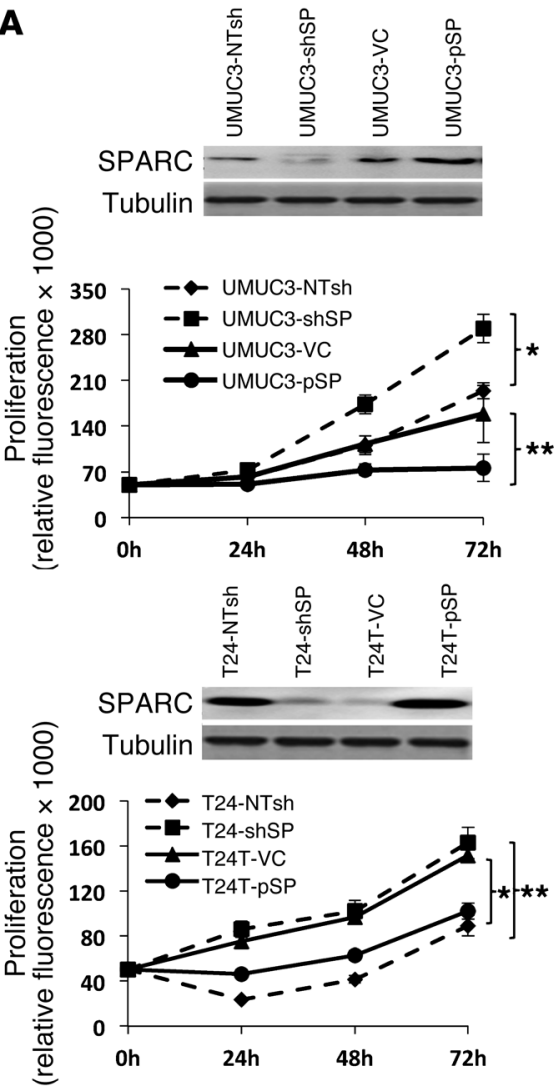

B
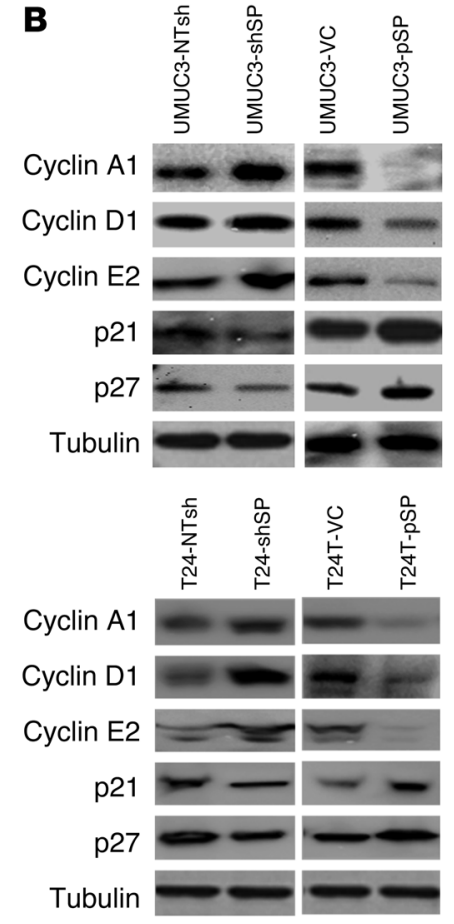

C
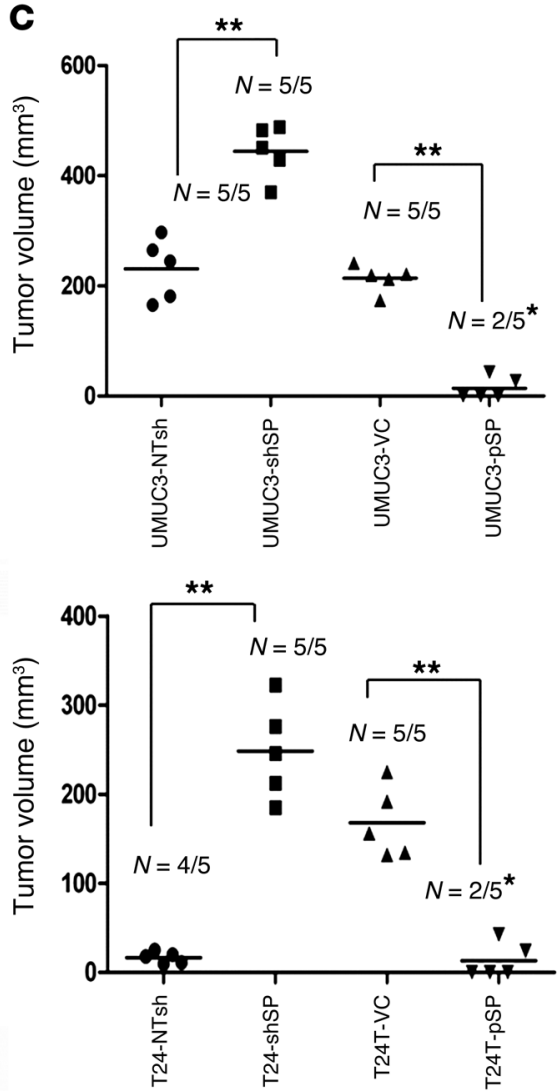

Figure 10

Effect of SPARC on UC cell proliferation in in vitro and in vivo tumorigenicity. (A) Western blots showing the expression of SPARC protein in $20 \mu \mathrm{g}$ protein of UMUC3 (top panel), T24, and T24T (bottom panel) human bladder cancer cell lines after overexpression and/or depletion of SPARC. The effect of genetic manipulation of SPARC on UC cell proliferation was determined by CyQuant assay. UMUC3, T24, and T24T transfected with full-length SPARC in pcDNA3.1 (pSP) and pcDNA3.1 empty vector control (VC) or transduced with lentiviral vector laden with short hairpin targeting Sparc gene (shSP) and its irrelevant nontarget control (NTsh). Representative growth curves of cell proliferation measured at 24, 48, and 72 hours performed twice in quadruplicate. ${ }^{*} P<0.05$, comparing cells overexpressing SPARC (pSP) with their matching VC; ${ }^{* *} P<0.05$, comparing cells depleted of SPARC with their matching NTsh, Student's $t$ test. (B) Western blot of cycle regulatory proteins cyclins A, D, and E and their inhibitors p21 and p27 in $20 \mu \mathrm{g}$ of the aforementioned cell lines. Equal protein loading was confirmed by reprobing blots with tubulin. (C) Scatter plots representing s.c. tumor take and volume of the UMUC3 T24, and T24T genetically manipulated for SPARC expression after injection in nude mice. ${ }^{*} P<0.05, \chi^{2}$ test; ${ }^{* *} P<0.05,2$-tailed Student's $t$ test.

cancerous compartment. Our findings regarding SPARC protein expression in the human bladder tumor and carcinogenesis model in the Sparc ${ }^{+/+}$mice may explain the apparently paradoxical reports in which expression of SPARC protein in cancer is decreased due to promoter methylation, while being overexpressed in the tumor associated stroma $(26,50-56)$. In our study, we show that SPARC protein expression increased in fibroblasts and macrophages in vitro in response to stimulation by cancer cells, the prototype ROS, and $\mathrm{H}_{2} \mathrm{O}_{2}$ as well as 8-isoprostane, another marker of oxidative tissue damage that further contributes to inflammation and ROS production. This observation is intriguing because the expression of SPARC has long been linked to physiological and pathological conditions characterized by extensive remodeling, desmoplasia, and plasticity, such as during embryonic development, wound healing, and cancer $(38,41,57-61)$, where it functions to maintain tissue homeostasis (summarized in refs. 10, 62).

Because tumors are viewed as "wounds that never heal" due to aberrant homeostasis (43), we speculate that the differential expression of SPARC in bladder tumors represents a state of aberrant homeostasis with inflammation that might be directly involved in urothelial cell transformation through the persistent progressive release of cytokines. Similarly, the enhanced in vivo growth of syngeneic tumors reported in Sparc ${ }^{-/-}$mice (summarized in ref. 38) and the growth of MB49 tumors reported herein highlight the role of SPARC in controlling many aspects of stromal desmoplasia, such as inflammation and angiogenesis supportive of tumor growth, invasiveness, and metastasis. Indeed, differentiation and phenotypic commitment of epithelial, mesenchymal, and neuronal cells require that cells exit cell cycle, arresting at $G_{0}-G_{1}$ / $S$ phase, and differentiate in response to microenvironmental cues (63). Since these programs are asynchronous and interconnected and oftentimes cells are locked in intermediate stages (summarized in ref. 63), it was not surprising to find increased SPARC levels in early stages of differentiation of growth-arrested nonproliferating fibroblasts and macrophages in response to inflammatory stimuli (Supplemental Figures 5 and 6) and hence, the lack of 
correlation of stromal expression of SPARC protein and/or cellcycle regulators with disease progression and DSS in murine and human tumor tissues.

Herein, we demonstrate that SPARC inhibited the acquisition inflammatory secretory phenotype of TAMs and CAFs through inhibition of the activation of NF-KB and AP- 1 in heterotypic cultures with cancer cells with subsequent decrease in their secreted cytokines and cancer cell invasiveness (Figure 7). Interestingly, among the secreted factors that were significantly downregulated in the presence of SPARC, TGF- $\beta$ and SDF1 were exclusively contributed by CAFs. Both TGF- $\beta$ and SDF1 have been implicated in the recruitment and differentiation of fibroblasts in different remodeling tissues (43) and were implicated in cancer cell invasiveness (reviewed in ref. 43). The reciprocal regulation of TGF- $\beta$ and SPARC has been reported in many physiological and pathological contexts $(20,38,64)$. Recent studies reported that in Sparc ${ }^{-1}$ pancreatic tumors, aberrant TGF- $\beta$ activation and high levels of TGF- $\beta$ were associated with decreased pericyte recruitment, destabilization of blood vessels $(42,65)$, vascular permeability, and inflammation contributing to tumor progression (17). Our results (Figures 6-8) indicate that SPARC attenuated the cancer-stromal cell crosstalk with subsequent inhibition of the inflammatory phenotype of fibroblasts and macrophages, as demonstrated by its differential effects on activation of NF-KB and AP- 1 in heterotypic cocultures. SPARC markedly inhibited the feed-forward loop that is reciprocated and maintained among cancer cells, TAMs, and CAFs through secreted inflammatory molecules, such as ROS, growth factors, and cytokines/chemokines that act as a double-edged sword in the tumor microenvironment. On the one hand, they sustain cancer cell proliferation, invasiveness, angiogenesis, and metastasis $(7,11$, $12,18)$. On the other hand, they play a critical role in recruitment and differentiation of stromal cells. The translational significance of this study stems from our findings of the inhibitory effect of tumor cell and host SPARC on urothelial carcinogenesis, progression, and metastasis (Supplemental Figure 11). Forced overexpression of SPARC in medulloblastoma, ovarian, and colorectal cancer cell lines reversed their resistance and enhanced their sensitivity to standard of care chemotherapy and radiation therapy $(50,66$, 67). In addition, the inverse relationship between SPARC tumor expression and the increased activation of NF-KB and AP-1 target molecules represents viable therapeutic targets. The upregulation of inflammatory cytokines and mediators can be targeted by small molecule inhibitors and neutralizing antibodies to retard metastatic disease. Of note is our report of the efficacy of targeting the CCL2/CCR2 axis in inhibiting lung metastases in models of bladder cancer metastasis (29). In view of our findings herein, the use of SPARC in urothelial cancer in the adjuvant and/or neoadjuvant settings as a single agent or in combination with agents that target downstream effectors is warranted.

\section{Methods}

Transgenic animal model. All animal protocols were approved by the ACUC of the University of Virginia. Sparc ${ }^{-/-}$mice were originally obtained from The Jackson Laboratory. Sparc/- mice were originally generated by targeted disruption of the Sparc locus in mouse ES cells by insertion of neomycinresistance genes into exon 4 of the Sparc gene derived from mouse strain $129 \mathrm{~Sv}$ and crossed with C57BL/6 mice as earlier detailed (68). Mice used in the present study were maintained in C57BL/6 background for at least 10 generations (16). Four-week-old Sparc ${ }^{-/}$and Sparc ${ }^{+/}$mice in a C57BL/6 background $(16,68)$ were started on $0.05 \% \mathrm{BBN}$ in drinking water. An initial study was conducted in which mice were monitored 3 times per week and surrogate end points of survival were as follows: impaired mobility, behavior, activity, blood on urethra, change in feeding/drinking habits, weight loss/cachexia $>20 \%$ of initial weight, and any sign of pain or distress not relieved by standard treatments. Mice were euthanized by isoflurane inhalation and cervical dislocation. In a second study, mice were euthanized at 8 weeks, $8-15$ weeks, $15-25$ weeks, and 25 weeks, and bladders were sagittally dissected into 2 halves and inspected for gross tumors. One half was processed for histopathologic examination and the other was snap-frozen in liquid nitrogen and stored at $-80^{\circ} \mathrm{C}$ for further studies. Three to five micron sections were stained with $\mathrm{H} \& \mathrm{E}$ and examined for tumor. Diagnoses were as follows: (a) normal, 3-7 cell layers with normal maturation from basal to luminal levels that includes large/flat umbrella cells; (b) IAD, nuclear (pleomorphism) or architectural distortion without increased number of cells (c) CIS, flat lesion within the urothelial layer displaying loss of polarity/differentiation with marked nuclear pleomorphism, high nuclear/ cytoplasmic ratio, and mitotic figures; and (d) invasive carcinoma, invasion was diagnosed if tumor cells were seen infiltrating the muscle layers of the bladder. Para-aortic LNs, kidneys, lungs, and liver were also dissected and grossly and microscopically examined for pathological changes. All slides were examined by a urologic pathologist (H.F. Frierson). Formalin-fixed, paraffin-embedded tissues were deparaffinized and stained with $\mathrm{H} \& \mathrm{E}$ or immunohistochemical (IHC) staining; see below.

Western blotting. Peeled urothelia at different time points were homogenized in $500 \mu$ l of lysis buffer $(50 \mathrm{mM}$ Tris, $\mathrm{pH} 7.5,100 \mathrm{mM} \mathrm{NaCl}, 5 \mathrm{mM}$ EDTA, 5 mM EGTA, 0.5\% NP-40, $40 \mathrm{mM} \beta$-glycerolphosphate, $0.5 \mu \mathrm{g} / \mathrm{ml}$ each leupeptin, pepstatin, and chymostatin, $50 \mathrm{mM} \mathrm{NaF}, 5 \mathrm{mM} \mathrm{Na}_{3} \mathrm{VO}_{4}$, with $1 \mu \mathrm{M}$ microcystin) for 10-20 minutes on ice. Protein concentrations were determined using a bicinchoninic acid (BCA) assay (Pierce). Equal amounts of cellular protein $(25 \mu \mathrm{g})$ were resolved on $4 \%-20 \%$ polyacrylamide gels and subsequently transferred on PVDF membrane (Bio-Rad). Monoclonal and polyclonal antibodies against mouse and human SPARC, human osteonectin, cyclin D1, cyclin E2, cyclin A, p21, p27, p65-NF-кB, phospho-p65-NF-kB, c-Jun, phospho-c-Jun, p38, phospho-p38, sulfiredoxin, NNMT, pancytokeratin, and $\alpha$-tubulin were purchased from R\&D Systems Inc., Haematologic Technologies, Invitrogen, Cell Signalling Technologies, Inc., Abcam, Millipore, and Santa Cruz Bitoechnology Corp., respectively. Chemiluminescence was detected using SuperSignal Femto Maximum Sensitivity Substrate (Pierce) (28) and quantified with FluoroChem 8800 (Alpha Innotech Corporation). Membranes were then stripped of antibodies and reprobed with monoclonal antibody against $\alpha$-tubulin to ensure equal protein loading.

8-isoprostane, 8-OH-dG, cytokine/growth factor assays. Tissue homogenates and $\mathrm{CM}$ from the experimental conditions described in the figure legends were collected and stored at $-80^{\circ} \mathrm{C}$. Commercial ELISA kits were used to determine the concentrations of IL-6, VEGF, TNF- $\alpha$, CCL2, TGF- $\beta$, SDF1, CCL3, and CXCL2 (RayBioTech. Inc. and R\&D Systems). Enzyme immunoassay (EIA) kits for 8-isoprostane and 8-hydroxy-2-deoxy Guanosine (8-OH-dG) were purchased from Cayman Chemicals Inc. and were used according to the manufacturer's instructions. For 8-OH-dG determination, DNA was extracted from tissue homogenates using a DNA extraction kit (QIAGEN) followed by digestion with P1 nuclease (Sigma-Aldrich) and treatment with Alkaline phosphatase (Sigma-Aldrich).

Isolation and short-term culture of primary urothelial cells, fibroblasts, and macrophages. Following resection, NU and UC cells and fibroblasts were isolated from Sparc ${ }^{-/-}$and Sparc ${ }^{+/+}$bladders as earlier described $(12,69)$ with some modifications. The inner layers of bladders were peeled off and minced into approximately $1-\mathrm{mm}^{3}$ pieces under sterile conditions, washed, and then resuspended in $10 \mathrm{ml}$ of dissociation buffer (Sigma-Aldrich) and incubated for 2 hours at $37^{\circ} \mathrm{C}$ with vigorous shaking. The resulting cell- 
tissue mixture was washed 3 times in fresh keratinocyte growth medium-2 (KGM-2) supplemented with KGM-2 bullet kit and growth factors (Clonetics KGM-2 and SingleQuot Suppl. Kit; Lonza). The remaining bladder tissues were minced and digested in collagenase/dispase (Sigma-Aldrich) for 4 hours at $37^{\circ} \mathrm{C}$ and then cultured in DMEM/F12 medium supplemented with $10 \%$ FBS, $100 \mu \mathrm{g} / \mathrm{ml}$ streptomycin, 50 units $/ \mathrm{ml}$ penicillin, and $1.5 \mathrm{~g} /$ $\mathrm{ml}$ fungizone until adherent fibroblast colonies became confluent. Epithelial and fibroblast phenotypes were confirmed by morphologic appearance and by Western blotting for pankeratin, vimentin, and $\alpha$-smooth muscle actin (from Cell Signaling and Santa Cruz Biotechnology Inc.) for cancer cells and fibroblasts, respectively. All experiments used primary normal (passages 2-3) and cancer urothelial cells (passages 2-4) and fibroblasts (passages 2-6). Fibroblast differentiation into CAFs was induced in vitro by treating $80 \%$ confluent monolayers with $0.1 \% \mathrm{H}_{2} \mathrm{O}_{2}$ and/or $10 \mathrm{nM} 8$-isoprostane (Cayman Chemicals Inc.). SPARC-depleted CM from 72-hour confluent monolayers of MB49 cells were prepared by preclearing CM with $1 \mu \mathrm{g}$ goat anti-mouse SPARC (R\&D Inc.) for 4 hours followed by protein A/G sepharose beads (Sigma-Aldrich) per the manufacturer's protocol. The cleared CM was filter sterilized and added to primary fibroblasts.

Isolation of Sparc ${ }^{+/}$and Sparc/- monocytes/macrophages. Monocytes were isolated by flushing bone marrow with sterile PBS and were further purified by attachment assay. Attached monocytes were induced to macrophage differentiation by adding $10 \mathrm{ng} / \mathrm{ml}$ of GM-CSF (Peprotech) for 5 days in RPMI 1640 supplemented with $2 \mathrm{mM}$ L-glutamine and 10\% FBS (70). Twenty-four hours before the experiments, GM-CSF was omitted from the medium. Primary monocytes/macrophages were used between passages 2-6.

Cell lines, culture, gene transfer, and NF- $\mathrm{K} B$ and AP-1 luciferase assays. All cell culture reagents were from Invitrogen. UMUC3, T24, T24T, U937, and MB49 cells were obtained from and maintained as recommended by ATCC (28). UMUC3, T24T, and U937 cells were transfected with pSPARC $(12,13)$ cloned in pcDNA3.1 or empty vector control using Fugene 6 (Roche Applied Science) according to the manufacturer's instructions. Stable selection in G418 was used in in vivo experiments. Stable knockdown of SPARC was done using shRNAs targeting SPARC 5'-CCGGCGGTTGTTCTTTCCTCACATTCTCGAGAATGTGAGGAAAGAACAACCGTTTTT-3' or nontarget shRNA (NTsh) control vector, cloned in pLKO.1-puro (Mission-TRC; Sigma-Aldrich) following the manufacturer's protocol. shRNA plasmids were packaged in $293 \mathrm{~T}$ cells by cotransfection with compatible packaging plasmids (Addgene). Culture supernatants containing the viral particles were collected 48 hours after transfection and filtered through $0.45-\mu \mathrm{m}$ filters (Fisher Scientific). UMUC3 and T24 cells were transduced with lentivirus-containing supernatants in the presence of $8 \mu \mathrm{g} / \mathrm{ml}$ Polybrene (Sigma-Aldrich) for 24 hours. Virus-containing medium was replaced with selection medium containing $1 \mu \mathrm{g} / \mathrm{ml}$ puromycin (Sigma-Aldrich) for 2 weeks. Cells with the most efficient knockdown were used in subsequent experiments. The activation of NF-KB and AP-1 promoters was determined in human and murine UC cells, CAFs, and macrophages as described earlier (28).

Intracellular ROS formation in cells and tissues. To examine accumulation of superoxide anions, cells and frozen bladder sections were incubated with 10 $\mu \mathrm{M}$ DHE (Molecular Probes) for 30 minutes at $37^{\circ} \mathrm{C}$, after which they were analyzed by fluorescent microscopy (Leica). Cellular oxidation in the bladder tissue was assayed by quantifying the fluorescence intensity at $A_{510} / A_{595}$ excitation/emission in 6 fields/section $(n=6 /$ group). Image analysis was performed using Image J software (http://rsbweb.nih.gov/ij/index.html). Alternatively, cells were loaded with $10 \mu \mathrm{M}$ of $2^{\prime}, 7^{\prime}$-dichlorofluorescein diacetate ( $\mathrm{H}_{2} \mathrm{DCFDA}$, for brevity DCF) (Molecular Probes), a marker of cellular oxidation by hydrogen peroxide $\mathrm{H}_{2} \mathrm{O}_{2}$. At the end of incubation, cells were washed in PBS and exposed to different stimuli for the indicated times. The intensity of DCF was measured at $\mathrm{A}_{485} / \mathrm{A}_{525}$ excitation/emission (33).
In vitro proliferation and microinvasion assays. Collage I from rat tail and reduced growth factor Matrigel were purchased from BD Biosciences. Unless otherwise stated, all other chemicals were purchased from Sigma-Aldrich or Thermo Fisher. CyQuant assay (Invitrogen) was used to determine cell proliferation and DNA content per the manufacturer's instructions. Chemoinvasion assay was carried out with 24 -well and 8 - $\mu \mathrm{m}$-pore-size polycarbonate filters (Costar; Thermo Fisher) as previously described $(7,11,18,28)$. For blocking assays, cells were incubated with $1 \mathrm{mM}$ NAC (stock in deionized water) for at least 1 hour at $37^{\circ} \mathrm{C}$, followed by the addition of the $10 \mu \mathrm{M}$ DCF; the intensity of the fluorescence was scanned continuously for 1 hour in a plate reader at excitation and emission $\mathrm{A}_{485} \mathrm{~nm}$ and $\mathrm{A}_{525} \mathrm{~nm}$. Experiments were repeated twice in quadruplicate.

In vivo tumor xenografts and experimental and spontaneous metastasis models. Female athymic $\left(n u^{+} / n u^{+}\right)$mice, 4-6 weeks of age (NCI), were treated after approval of our experimental protocols by the Animal Care and Use Committee of the University of Virginia. The s.c. tumors were generated by injecting T24, T24T, and UMUC 3 cells $\left(10^{6}\right.$ cells $/ 0.1 \mathrm{ml}$ phenol red-free medium) s.c. into the flanks of 5 -week-old nude mice. Animals were monitored twice weekly for tumor development as described (16). For syngeneic models, Sparc ${ }^{-/}$and Sparc ${ }^{+/+}$mice were injected s.c. in 1 flank with $1 \times 10^{4} \mathrm{MB} 49$ cells/100 $\mu \mathrm{l}$ phenol red-free RPMI 1640. Animal survival and the development of s.c. tumors and lung metastasis were monitored. At the end of 14 days, lungs were dissected and processed as described earlier $(16,28)$. For experimental metastasis assays, mice received injections via lateral tail vein. Mice were euthanized 4 or 6 weeks after injection, lungs were harvested, and the number of visible surface metastases was determined. Tissues were either processed for immunostaining or snap-frozen for molecular analyses (28). For early metastasis efficiency assays, MB49 cells overexpressing GFP via retroviral transduction $\left(1 \times 10^{4}\right.$ cells $)$ and UMUC3-NTsh and shSPARC cells $\left(1 \times 10^{6}\right.$ cells $)$ labeled with CellTracker Green CMFDA $(10 \mu \mathrm{M}$; Molecular Probes) were i.v. injected via tail veins of $\mathrm{Sparc}^{-/-}$and $\mathrm{Sparc}^{+/+}$mice and nude mice, respectively (28). After 24, 48, and 72 hours, lungs were dissected, perfused with $10 \%$ neutral buffered formalin in PBS, and then fixed in OCT and processed for cryosectioning. The numbers of fluorescent tumor cells adherent to the lung vasculature and parenchyma were counted in 5 random fields of $10-\mu \mathrm{m}$ sections $(n=4$ animals) under fluorescent microscopy $\left(\mathrm{A}_{492} / \mathrm{A}_{517}\right.$, excitation/emission). Fluorescent images were analyzed by ImageJ software.

Construction and immunostaining of bladder cancer tissues. Protein expression pattern of human SPARC was assessed using 2 TMAs $(28,29)$. Antigen-retrieval methods $(0.01 \%$ citric acid for 15 minutes under microwave treatment) were utilized prior to incubation with mouse monoclonal anti-SPARC antibody (R\&D Systems Inc.) at 1:500. Staining conditions were optimized on sections from formalin-fixed, paraffin-embedded ovaries and testes as positive control, as recommended by the manufacturer. The absence of primary antibody was used as a negative control. The secondary antibodies (Vector Laboratories Inc.) were biotinylated horse antimouse antibodies (1:500 dilution). Diaminobenzidine was utilized as the final chromogen and hematoxylin as the nuclear counterstain $(28,29)$. The consensus value of the 3 or 4 representative cores from each tumor sample arrayed was used for statistical analyses. SPARC expression was evaluated in the tumor and in the stroma. SPARC "score" was the number of cells expressing the protein in the cytoplasm and nuclei. SPARC staining "intensity" was categorized as negative $(-)$, low (+), intermediate $(++)$, and high $(++)$. Tumor and stroma were evaluated separately. Cutoffs of expression for prognostic evaluation were selected based on the median values of expression among the groups under analyses. "High" score was staining of more than $10 \%$ of cells. "High" intensity was equal to or higher than "low" (+). 
Immunostaining of mouse tumor tissues was performed with Vectastain ABC ELITE or a MOMA Kit (Vector Laboratories, Inc.) according to manufacturer's instructions and counterstained with hematoxylin. Antigen retrieval was achieved as described above. We used monoclonal and polyclonal antibodies against the following: phospho-p65-NF-кB, phospho-c-Jun (Cell Signaling), Ki67 (Dako Cytomation), mac1 and CD31 (eBioscience), and goat anti-mouse SPARC (R\&D Systems Inc.). Scoring of the expression of phospho-p65-NF- $\mathrm{BB}$, and phospho-c-Jun in the cytoplasm and nuclei of urothelial (normal and cancerous) and stromal cells was carried out using a composite expression score (CES) combining the frequency and intensity of staining. The frequency of positive staining was determined by the number of positive cells per 100 cells counted in 6 independent fields/bladder section. Frequency score was set based on the percentage of positive cells: $10 \%$ (score 0$) ;+, 11 \%-40 \%(+), 41 \%-70 \%(++)$, and more than $70 \%(+++)$. The intensity of staining intensity score was as described above. For each section, a CES was generated by transforming the frequency and intensity scores into numeric values. This scoring system allows compensation for tumor heterogeneity as well as the sequential phosphorylation and nuclear translocation of these transcription factors. In all immunostaining experiments, negative controls were performed omitting the primary antibodies. In SPARC immunostaining, the specificity of the antibodies was validated by including recombinant human and murine SPARC (R\&D Systems Inc.) during staining to competitively inhibit the reaction of the antibodies with tissue SPARC (Supplemental Figure 12).

Statistics. The relationships among tumor histology, genotype, and age were assessed by applying the $\chi^{2}$ test. All other comparisons between $\mathrm{Sparc}^{-1-}$ and $\mathrm{Sparc}^{+/+}$groups were performed with 2-tailed Student's $t$ test. The changes of the levels of cytokines/chemokines, growth factors, and inflammatory mediators as a function of tumor progression in either $\mathrm{Sparc}^{-1-}$ or Sparc $\mathrm{Pumors}^{+/+}$were analyzed by 1-way ANOVA. Analyses were carried out with GraphPad Prism 5.0 software and Microsoft Excel. Com- parisons were considered significant at $P<0.05$. Statistical analysis of the CES of SPARC, phospho-p65-NF-kB, and phospho-c-Jun in mouse tissue was performed using Kruskal-Wallis 1-way ANOVA followed by Dunn's and Bonferroni's multiple comparison post-hoc analyses (GraphPad Prism 5.0). Associations of SPARC expression in TMAs with DSS were evaluated using the log-rank test. DSS time was equivalent to months between transurethral resection or cystectomy and death of disease. Survival plotted using Kaplan-Meier methodology (SPSS) $(28,29)$

Study approval. All animal experiments were performed after approval of protocol and in compliance with guidelines of the Animal Care and Use Committee of the University of Virginia. Bladder cancer TMAs were constructed at the Spanish National Cancer Institute $(28,29)$. These arrays included primary urothelial cell carcinomas of the bladder belonging to patients recruited under Institutional Review Board-approved protocols; patients gave informed consent in studies referenced $(28,29)$.

\section{Acknowledgments}

This study was supported by NIH grant CA143971 (to D. Theodorescu), the Paul Mellon Urologic Cancer Institute, University of Virginia (to N. Said), and CA118240 (to R.A. Brekken). The authors wish to thank Sharon Birdsall, John Sanders, Melissa Bevard, and Jeremy Gatesman for technical help with experiments.

Received for publication May 14, 2012, and accepted in revised form November 8, 2012.

Address correspondence to: Dan Theodorescu, Departments of Surgery and Pharmacology and Comprehensive Cancer Center, University of Colorado, Aurora, Colorado 80045, USA. Phone: 303.724.7135; Fax: 303.724.3162; E-mail: dan.theodorescu@ ucdenver.edu.
1. Jemal A, Bray F, Center MM, Ferlay J, Ward E, Forman D. Global cancer statistics. CA Cancer J Clin. 2011;61(2):69-90.

2. Siegel R, Naishadham D, Jemal A. Cancer statistics, 2012. CA Cancer J Clin. 2012;62(1):10-29.

3. Iida $\mathrm{K}$, et al. Nrf2 and p53 cooperatively protect against $\mathrm{BBN}$-induced urinary bladder carcinogenesis. Carcinogenesis. 2007;28(11):2398-2403.

4. Karin M. Nuclear factor-kappaB in cancer development and progression. Nature. 2006; 441(7092):431-436.

5. Sakurai T, Maeda S, Chang L, Karin M. Loss of hepatic NF-kappa B activity enhances chemical hepatocarcinogenesis through sustained c-Jun N-terminal kinase 1 activation. Proc Natl Acad Sci US A. 2006;103(28):10544-10551.

6. He G, Karin M. NF-kappaB and STAT3 - key players in liver inflammation and cancer. Cell Res. 2011;21(1):159-168.

7. Said NA, Elmarakby AA, Imig JD, Fulton DJ, Motamed K. SPARC ameliorates ovarian cancer-associated inflammation. Neoplasia. 2008;10(10):1092-1104.

8. Arnold SA, et al. Lack of host SPARC enhances vascular function and tumor spread in an orthotopic murine model of pancreatic carcinoma. Dis Model Mech. 2010;3(1-2):57-72.

9. Chlenski A, Cohn SL. Modulation of matrix remodeling by SPARC in neoplastic progression. Semin Cell Dev Biol. 2010;21(1):55-65.

10. Chiodoni C, Colombo M, Sangaletti S. Matricellular proteins: from homeostasis to inflammation, cancer, and metastasis. Cancer and Metastasis Reviews. 2010;29(2):295-307.

11. Said N, Socha MJ, Olearczyk JJ, Elmarakby AA, Imig JD, Motamed K. Normalization of the ovarian cancer microenvironment by SPARC. Mol Cancer Res. 2007;5(10):1015-1030.
12. Said NA, Najwer I, Socha MJ, Fulton DJ, Mok SC, Motamed K. SPARC inhibits LPA-mediated mesothelial-ovarian cancer cell crosstalk. Neoplasia. 2007;9(1):23-35.

13. Said N, Frierson HF Jr, Chernauskas D, Conaway M, Motamed K, Theodorescu D. The role of SPARC in the TRAMP model of prostate carcinogenesis and progression. Oncogene. 2009;28(39):3487-3498.

14. Said N, Theodorescu D. Pathways of metastasis suppression in bladder cancer. Cancer Metastasis Rev. 2009;28(3-4):327-333.

15. Sangaletti S, et al. Macrophage-derived SPARC bridges tumor cell-extracellular matrix interactions toward metastasis. Cancer Res. 2008;68(21):9050-9059.

16. Said N, Motamed K. Absence of host-secreted protein acidic and rich in cysteine (SPARC) augments peritoneal ovarian carcinomatosis. Am J Pathol. 2005;167(6):1739-1752.

17. Arnold SA, et al. Losartan slows pancreatic tumor progression and extends survival of SPARC-null mice by abrogating aberrant TGFbeta activation. PloS One. 2012;7(2):e31384.

18. Said N, Najwer I, Motamed K. Secreted protein acidic and rich in cysteine (SPARC) inhibits integrinmediated adhesion and growth factor-dependent survival signaling in ovarian cancer. Am J Pathol. 2007;170(3):1054-1063.

19. Chlenski A, et al. Anti-angiogenic SPARC peptides inhibit progression of neuroblastoma tumors. $\mathrm{Mol}$ Cancer. 2010;9:138.

20. Bassuk JA, et al. Induction of TGF-beta1 by the matricellular protein SPARC in a rat model of glomerulonephritis. Kidney Int. 2000;57(1):117-128.

21. Gooden MD, Vernon RB, Bassuk JA, Sage EH. Cell cycle-dependent nuclear location of the matricellular protein SPARC: association with the nuclear matrix. J Cell Biochem. 1999;74(2):152-167.
22. Hudson AE, Feng WC, Delostrinos CF, Carmean N, Bassuk JA. Spreading of embryologically distinct urothelial cells is inhibited by SPARC.J Cell Physiol. 2005;202(2):453-463.

23. Kosman J, Carmean N, Leaf EM, Dyamenahalli K, Bassuk JA. The motif of SPARC that inhibits DNA synthesis is not a nuclear localization signal. $\mathrm{J} \mathrm{Mol}$ Biol. 2007;371(4):883-901.

24. Yamanaka M, et al. Analysis of the gene expression of SPARC and its prognostic value for bladder cancer. J Urol. 2001;166(6):2495-2499.

25. Nimphius W, Moll R, Olbert P, Ramaswamy A, Barth PJ. CD34+ fibrocytes in chronic cystitis and noninvasive and invasive urothelial carcinomas of the urinary bladder. Virchows Arch. 2007;450(2):179-185.

26. Larson J, et al. SPARC gene expression is repressed in human urothelial cells (UROtsa) exposed to or malignantly transformed by cadmium or arsenite. Toxicol Lett. 2010;199(2):166-172.

27. Gildea JJ, et al. RhoGDI2 is an invasion and metastasis suppressor gene in human cancer. Cancer Res. 2002;62:6418-6423.

28. Said N, Smith S, Sanchez-Carbayo M, Theodorescu D. Tumor endothelin-1 enhances metastatic colonization of the lung in mouse xenograft models of bladder cancer. J Clin Invest. 2011;121(1):132-147.

29. Said N, Smith S, Sanchez-Carbayo M, Theodorescu D. RhoGDI2 suppresses lung metastasis in mice by reducing tumor versican expression and macrophage infiltration. J Clin Invest. 2012;122(4):1503-1518.

30. Williams PD, Lee JK, Theodorescu D. Molecular credentialing of rodent bladder carcinogenesis models. Neoplasia. 2008;10(8):838-846.

31. He G, et al. Hepatocyte IKKbeta/NF-kappaB inhibits tumor promotion and progression by preventing oxidative stress-driven STAT3 activation. Cancer Cell. 2010;17(3):286-297. 
32. Huang YJ, Zhang BB, Ma N, Murata M, Tang AZ, Huang GW. Nitrative and oxidative DNA damage as potential survival biomarkers for nasopharyngeal carcinoma. Med Oncol. 2010;28(1):377-384.

33. Socha MJ, Manhiani M, Said N, Imig JD, Motamed K. Secreted protein acidic and rich in cysteine deficiency ameliorates renal inflammation and fibrosis in angiotensin hypertension. Am J Pathol. 2007;171(4):1104-1112.

34. Wei Q, et al. Sulfiredoxin-Peroxiredoxin IV axis promotes human lung cancer progression through modulation of specific phosphokinase signaling. Proc Natl Acad Sci U S A. 2011;108(17):7004-7009.

35. Yin Y, et al. JNK/AP-1 pathway is involved in tumor necrosis factor-alpha induced expression of vascular endothelial growth factor in MCF7 cells. Biomed Pharmacother. 2009;63(6):429-435.

36. Wu Y, Siadaty MS, Berens ME, Hampton GM, Theodorescu D. Overlapping gene expression profiles of cell migration and tumor invasion in human bladder cancer identify metallothionein $1 \mathrm{E}$ and nicotinamide $\mathrm{N}$-methyltransferase as novel regulators of cell migration. Oncogene. 2008;27(52):6679-6689.

37. Wei Q, Jiang H, Matthews CP, Colburn NH. Sulfiredoxin is an AP- 1 target gene that is required for transformation and shows elevated expression in human skin malignancies. Proc Natl Acad Sci U S A. 2008;105(50):19738-19743.

38. Sangaletti S, et al. SPARC oppositely regulates inflammation and fibrosis in bleomycin-induced lung damage. Am J Pathol. 2011;179(6):3000-3010.

39. Castello-Cros R, Cukierman E. Stromagenesis during tumorigenesis: characterization of tumor-associated fibroblasts and stroma-derived 3D matrices. Methods Mol Biol. 2009;522:275-305.

40. Bradshaw AD, Reed MJ, Carbon JG, Pinney E, Brekken RA, Sage EH. Increased fibrovascular invasion of subcutaneous polyvinyl alcohol sponges in SPARCnull mice. Wound Repair Regen. 2001;9(6):522-530.

41. Chlenski A, et al. SPARC enhances tumor stroma formation and prevents fibroblast activation. Oncogene. 2007;26(31):4513-4522.

42. Rivera LB, Brekken RA. SPARC promotes pericyte recruitment via inhibition of endoglin-dependent TGF-beta1 activity. J Cell Biol. 2011;193(7):1305-1319.

43. Cirri P, Chiarugi P. Cancer-associated-fibroblasts and tumour cells: a diabolic liaison driving cancer progression. Cancer Metastasis Rev. 2012;31(1-2):195-208.

44. Takahashi H, Ogata H, Nishigaki R, Broide DH, Karin M. Tobacco smoke promotes lung tumorigenesis by triggering IKKbeta- and JNK1-depen- dent inflammation. Cancer Cell. 2010;17(1):89-97.

45. Aksoy S, Szumlanski CL, Weinshilboum RM. Human liver nicotinamide $\mathrm{N}$-methyltransferase. cDNA cloning, expression, and biochemical characterization. J Biol Chem. 1994;269(20):14835-14840.

46. Tang S-W, et al. Nicotinamide N-methyltransferase induces cellular invasion through activating matrix metalloproteinase- 2 expression in clear cell renal cell carcinoma cells. Carcinogenesis. 2010;32(2):138-145.

47. Naugler WE, Karin M. NF-kappaB and canceridentifying targets and mechanisms. Curr Opin Genet Dev. 2008;18(1):19-26.

48. Sakurai T, et al. Hepatocyte necrosis induced by oxidative stress and IL-1 alpha release mediate carcinogen-induced compensatory proliferation and liver tumorigenesis. Cancer Cell. 2008;14(2):156-165.

49. Shibata W, et al. c-Jun NH2-terminal kinase 1 is a critical regulator for the development of gastric cancer in mice. Cancer Res. 2008;68(13):5031-5039.

50. Cheetham S, Tang MJ, Mesak F, Kennecke H, Owen D, Tai IT. SPARC promoter hypermethylation in colorectal cancers can be reversed by 5-Aza-2' deoxycytidine to increase SPARC expression and improve therapy response. BrJCancer. 2008;98(11):1810-1819.

51. DiMartino JF, et al. Low or absent SPARC expression in acute myeloid leukemia with MLL rearrangements is associated with sensitivity to growth inhibition by exogenous SPARC protein. Leukemia: official journal of the Leukemia Society of America; Leukemia Research Fund, U.K. 2006;20(3):426-432.

52 . Gao J, et al. Methylation of the SPARC gene promoter and its clinical implication in pancreatic cancer. J Exp Clin Cancer Res. 2010;29:28.

53. Rodriguez-Jimenez FJ, Caldes T, Iniesta P, Vidart JA, Garcia-Asenjo JL, Benito M. Overexpression of SPARC protein contrasts with its transcriptional silencing by aberrant hypermethylation of SPARC CpG-rich region in endometrial carcinoma. Oncol Rep. 2007;17(6):1301-1307.

54. Socha MJ, et al. Aberrant promoter methylation of SPARC in ovarian cancer. Neoplasia. 2009;11(2):126-135.

55. Yang E, Kang HJ, Koh KH, Rhee H, Kim NK, Kim $\mathrm{H}$. Frequent inactivation of SPARC by promoter hypermethylation in colon cancers. Int J Cancer. 2007;121(3):567-575.

56. Yoshimura T, Nagahara M, Kuo C, Turner RR, Soon-Shiong P, Hoon DS. Lymphovascular invasion of colorectal cancer is correlated to SPARC expression in the tumor stromal microenvironment. Epigenetics. 2011;6(8):1001-1011.
57. Bradshaw AD, Francki A, Motamed K, Howe C, Sage EH. Primary mesenchymal cells isolated from SPARCnull mice exhibit altered morphology and rates of proliferation. Mol Biol Cell. 1999;10(5):1569-1579.

58. Bradshaw AD, Reed MJ, Sage EH. SPARC-null mice exhibit accelerated cutaneous wound closure. J Histochem Cytochem. 2002;50(1):1-10.

59. Rentz TJ, Poobalarahi F, Bornstein P, Sage EH, Bradshaw AD. SPARC regulates processing of procollagen I and collagen fibrillogenesis in dermal fibroblasts. J Biol Chem. 2007;282(30):22062-22071.

60. Nie J, Bradshaw AD, Delany AM, Sage EH. Inactivation of SPARC enhances high-fat diet-induced obesity in mice. Connect Tissue Res. 2011;52(2):99-108.

61. Nie J, Sage EH. SPARC inhibits adipogenesis by its enhancement of beta-catenin signaling.J Biol Chem. 2009;284(2):1279-1290.

62. Rivera LB, Bradshaw AD, Brekken RA. The regulatory function of SPARC in vascular biology. Cell Mol Life Sci. 2011;68(19):3165-3173.

63. Strauss R, Hamerlik P, Lieber A, Bartek J. Regulation of stem cell plasticity: mechanisms and relevance to tissue biology and cancer. Mol Ther. 2012;20(5):887-897.

64. Atorrasagasti C, et al. SPARC downregulation attenuates the profibrogenic response of hepatic stellate cells induced by TGF-beta1 and PDGF. Am J Physiol Gastrointest Liver Physiol. 2011;300(5):G739-G748.

65. Parvani JG, Taylor MA, Schiemann WP. Noncanonical TGF-beta signaling during mammary tumorigenesis. J Mammary Gland Biol Neoplasia. 2011;16(2):127-146.

66. Chetty C, Dontula R, Gujrati M, Dinh DH, Lakka SS. Blockade of SOX4 mediated DNA repair by SPARC enhances radioresponse in medulloblastoma. Cancer Lett. 2012;323(2):188-198.

67. Bull Phelps SL, et al. Secreted protein acidic and rich in cysteine as a regulator of murine ovarian cancer growth and chemosensitivity. Am J Obstet Gynecol. 2009;200(2):180 e181-e187.

68. Norose K, et al. SPARC deficiency leads to earlyonset cataractogenesis. Invest Ophthalmol Vis Sci. 1998;39(13):2674-2680.

69. Roberson KM, et al. Isolation and characterization of a novel human bladder cancer cell line: BK10. In Vitro Cell Dev Biol Anim. 1998;34(7):537-544.

70. Sangaletti S, Stoppacciaro A, Guiducci C, Torrisi MR, Colombo MP. Leukocyte, rather than tumorproduced SPARC, determines stroma and collagen type IV deposition in mammary carcinoma. J Exp Med. 2003;198(10):1475-1485. 